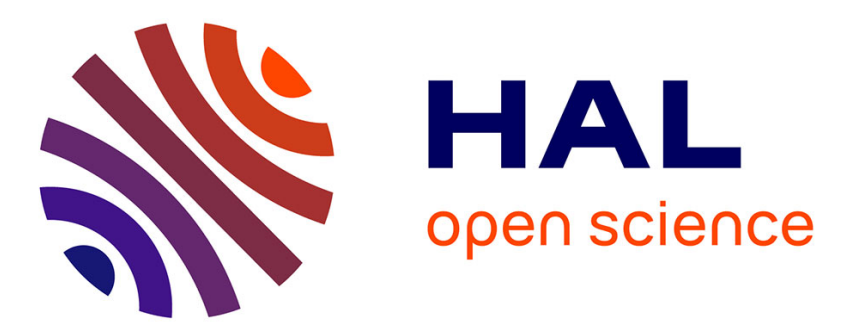

\title{
A novel ankle rehabilitation device based on a reconfigurable 3-RPS parallel manipulator
}

Latifah Nurahmi, Stéphane Caro, Mochamad Solichin

\section{To cite this version:}

Latifah Nurahmi, Stéphane Caro, Mochamad Solichin. A novel ankle rehabilitation device based on a reconfigurable 3-RPS parallel manipulator. Mechanism and Machine Theory, 2019, 134, pp.135-150. 10.1016/j.mechmachtheory.2018.12.017 . hal-01966590

\section{HAL Id: hal-01966590 \\ https://hal.science/hal-01966590}

Submitted on 28 Dec 2018

HAL is a multi-disciplinary open access archive for the deposit and dissemination of scientific research documents, whether they are published or not. The documents may come from teaching and research institutions in France or abroad, or from public or private research centers.
L'archive ouverte pluridisciplinaire HAL, est destinée au dépôt et à la diffusion de documents scientifiques de niveau recherche, publiés ou non, émanant des établissements d'enseignement et de recherche français ou étrangers, des laboratoires publics ou privés. 


\title{
A Novel Ankle Rehabilitation Device based on a Reconfigurable 3-RPS Parallel Manipulator
}

\author{
Latifah Nurahmi ${ }^{\star}$ \\ latifah.nurahmi@me.its.ac.id \\ Stéphane Caro $^{\dagger}$ \\ Mochamad Solichin ${ }^{\ddagger}$ \\ stephane.caro@ls $2 \mathrm{n} . \mathrm{fr}$ \\ solichin@me.its.ac.id

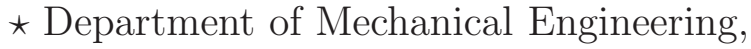 \\ Institut Teknologi Sepuluh Nopember, \\ Kampus ITS Sukolilo, 60111, Surabaya, Indonesia \\ $\dagger$ Centre National de la Recherche Scientifique, \\ Laboratoire des Sciences du Numérique de Nantes, \\ UMR CNRS 6004, 1 Rue de la Noë, 44321 Nantes, France
}

\begin{abstract}
This paper deals with the constraint-based design, optimum design and reconfiguration strategy of a 3-RPS parallel manipulator. Some design conditions related to the base and moving-platform design parameters and the three degree of-freedom operation modes are derived. A 3-RPS parallel manipulator with two types of operation modes is generated by following those conditions. Due to its potential advantages, this manipulator is used as an ankle rehabilitation device which can cover the ankle joint motion. To derive the optimum parameters, kinematic optimization is conducted by initially parametrizing the orientation workspace and it turns out that its orientation workspace is not symmetrical. The singularity loci are traced in its orientation workspace. A performance index, named Maximum Inscribed Circle Diameter (MICD) is presented to assess the maximum tilt of the moving-platform for any azimuth angle. The distributions of MICD are plotted in the design space for different moving-platform heights. The optimum region with regard to MICD is obtained. It is noteworthy that the evolution of MICD as a function of movingplatform height in both operation modes is the opposite. Therefore, a reconfiguration strategy is proposed to ensure the moving-platform working above the minimum required orientation for any moving-platform height.
\end{abstract}

Keywords: Design conditions, optimization, workspace analysis, operation modes, reconfiguration, singularities. 


\section{Introduction}

The well-known 3-RPS (R, P, S denote revolute, prismatic, spherical joints, respectively) parallel manipulator with different arrangements of RPS legs has been extensively studied by many researchers. In 1983 [1], the 3-RPS parallel manipulator with equilateral base and platform was introduced by Hunt. Schadlbauer et al. in [2] used algebraic approaches explained in [3, 4, 5], to enumerate sixteen solutions for the direct kinematics of this manipulator. The axode presented in [6] was employed by Schadlbauer et al. in [7] to characterize two operation modes belonging to the 3-RPS parallel manipulator. Modified 3-RPS parallel manipulator was introduced by Gan et al. in [8] where the manipulator can be reconfigured into the 3-RPS Cube. The kinematic behaviour of the 3-RPS Cube parallel manipulator was discussed in $[9,10,11]$.

Kalla et al. studied the $\Sigma^{2}$ singularities of the 3-RPS parallel manipulator [12]. Interchanging the moving-platform with the fixed base in the 3-RPS parallel manipulator, results in the 3-SPR parallel manipulator. Nayak et al. compared the singularity-free workspace of the 3-RPS and 3-SPR manipulators by means of Maximum Inscribed Circle Radius (MICR) [13]. MICR was also employed in [14] to compare operation modes and singularities of the 3-PRS parallel manipulator.

The dimension synthesis of parallel manipulators with multiple operation modes can be assessed by its orientation capability $[15,16,17]$ through its singularity loci since many applications take advantage of rotations. Several performance indices were proposed by researchers to measure how well the manipulators behave with regard to external wrenches and motions. Several transmission indices have been proposed in the literature, e.g., the virtual coefficient [18], the transmission index (TI) [19], the generalized transmission index (GTI) [20], the output transmission index (OTI) and the constraint transmission index (CTI) [21, 22], good transmission orientational workspace (GTOW) [23], variable motion/force transmissibility [24].

This paper focuses on the constraint-based design and dimension synthesis of a 3-RPS parallel manipulator for ankle rehabilitation [25, 26, 27]. The 3-RPS parallel manipulator is generated by following the design conditons derived in this paper. The three revolute joint axes intersect at one point. For any value of design parameters, the 3-RPS parallel manipulator always has two 3-dof operation modes, namely $x_{1}=0$ and $x_{2}=0$. These two operation modes differ from the ones of the 3-RPS parallel manipulator discussed in [2]. The orientation workspace is parametrized to recognize the physical interpretations of rotational motions. The singularities are investigated and a performance index, named Maximum Inscribed Circle Diameter (MICD), is proposed. This index is used for the dimension synthesis of the manipulator studied in this paper. The reconfiguration strategy is presented to ensure that the manipulator can work at minimum required orientation for any moving-platform height.

This paper is organized as follows: a detailed description of the manipulator architecture and its targeted application for ankle rehabilitation are defined in Section 2. Some design conditions are given in Section 3. The orientation workspace and singularities of the manipulator are analysed in Section 4. The dimension synthesis problem is formulated and solved in Section 5. Eventually, Section 6 presents a reconfiguration strategy for the manipulator to work at minimum required orientation for any moving-platform height. 


\section{Manipulator architecture and application}

\subsection{Manipulator architecture}

The architecture of 3-RPS parallel manipulator studied in this paper is shown in Fig. 1. This manipulator has three RPS legs in which each RPS leg consists of a revolute joint, a prismatic joint and a spherical joint. The three revolute joint axes intersect at point $A_{1}$.

The $(x, y, z)$ coordinate system of origin $O$ is defined as the fixed frame $\Sigma_{0}$ and located at the base. The base is bounded by the three revolute joints. The $i$-th revolute joint axis passing through point $A_{i}$ and its unit vector is $\mathbf{s}_{i}(i=1,2,3)$. Point $A_{i}$ can be located anywhere in the plane $z=0$ depending upon the design parameters $a_{i}$ and $\varepsilon_{i} . a_{i}$ is the distance between points $O$ and $A_{i}$, and $\varepsilon_{i}$ is the angle between $x$-axis and vector $\overline{O A_{i}}$. In what remains, sine, cosine and tangent of angle $\varepsilon_{i}$ is written as $s_{\varepsilon_{i}}=\sin \left(\varepsilon_{i}\right), c_{\varepsilon_{i}}=\cos \left(\varepsilon_{i}\right)$ and $t_{\varepsilon_{i}}=\tan \left(\varepsilon_{i}\right)$.

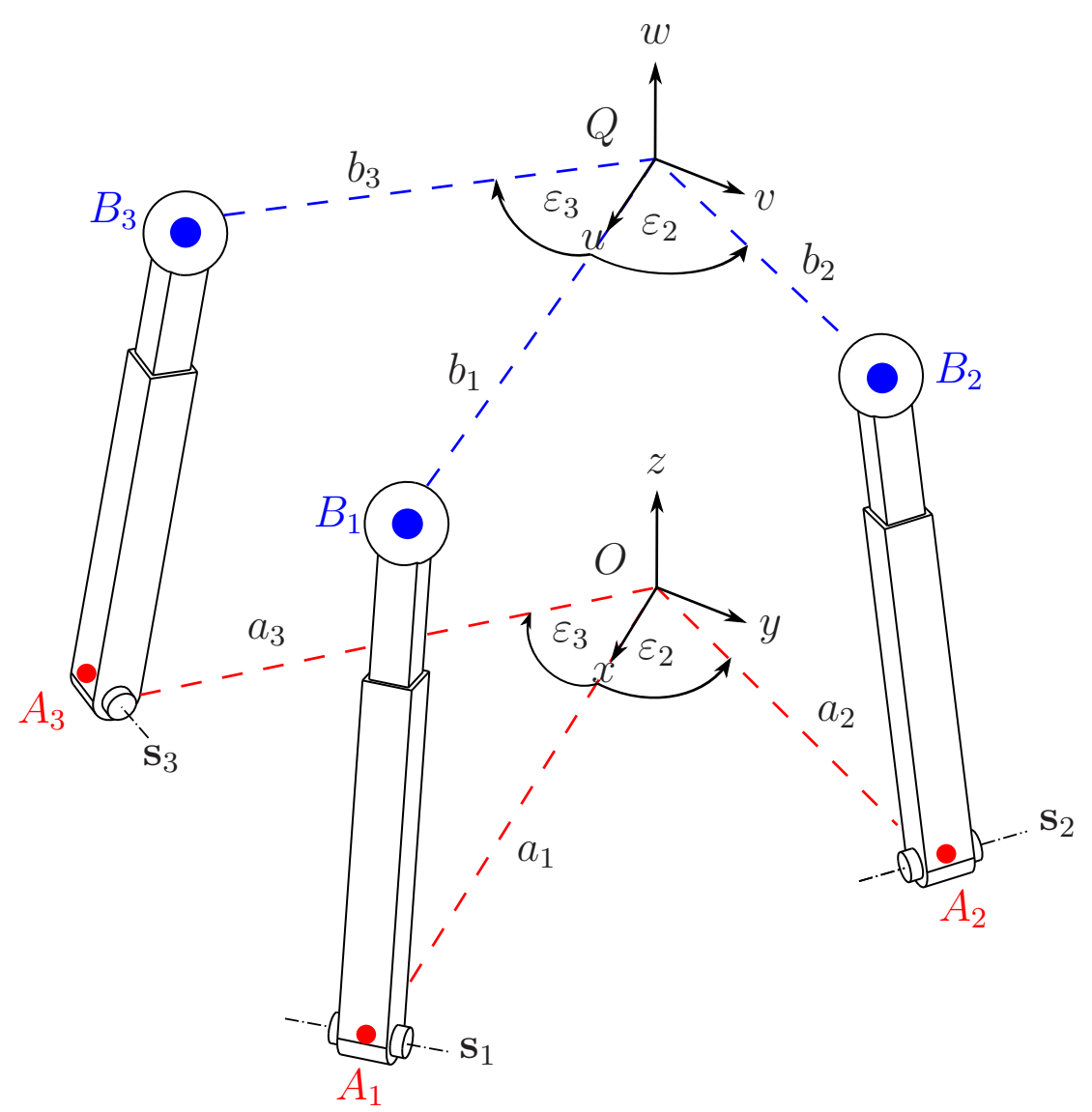

Figure 1: Parametrization of the 3-RPS parallel manipulator.

In the following, we use projective coordinates to define the position vectors of point $A_{i}$, $i=1,2,3$. Point $A_{1}$ is assumed to be located on the $x$-axis, hence $\varepsilon_{1}=0^{\circ}$ and the coordinates of points $A_{i}$ and vector $\mathbf{s}_{i}$ expressed in $\Sigma_{0}$ are: 


$$
\begin{gathered}
\mathbf{r}_{A_{1}}^{0}=\left[\begin{array}{c}
1 \\
a_{1} \\
0 \\
0
\end{array}\right], \quad \mathbf{r}_{A_{2}}^{0}=\left[\begin{array}{c}
1 \\
c_{\varepsilon_{2}} a_{2} \\
s_{\varepsilon_{2}} a_{2} \\
0
\end{array}\right], \quad \mathbf{r}_{A_{3}}^{0}=\left[\begin{array}{c}
1 \\
c_{\varepsilon_{3}} a_{3} \\
s_{\varepsilon_{3}} a_{3} \\
0
\end{array}\right], \\
\mathbf{s}_{1}=\left[\begin{array}{l}
0 \\
0 \\
1 \\
0
\end{array}\right], \quad \mathbf{s}_{2}=\left[\begin{array}{c}
0 \\
-s_{\varepsilon_{2}} \\
c_{\varepsilon_{2}} \\
0
\end{array}\right], \quad \mathbf{s}_{3}=\left[\begin{array}{c}
0 \\
-s_{\varepsilon_{3}} \\
c_{\varepsilon_{3}} \\
0
\end{array}\right] .
\end{gathered}
$$

The $(u, v, w)$ coordinate system of origin $Q$ is named $\Sigma_{1}$ and attached to the movingplatform. Three spherical joints are located at the vertices of the moving-platform. The center of the $i$-th spherical joint is denoted by $B_{i}(i=1,2,3)$. The locations of point $B_{i}$ can be anywhere on the plane $w=0$ depending upon the design parameters $b_{i}$ and $\varepsilon_{i}$, in which $b_{i}$ is the distance between points $B_{i}$ and $Q$. The first point $B_{1}$ is assumed to be located on the $u$-axis, hence $\varepsilon_{1}=0^{\circ}$. The coordinates of points $B_{i}$ are expressed in $\Sigma_{1}$ as follows:

$$
\mathbf{r}_{B_{1}}^{1}=\left[\begin{array}{c}
1 \\
b_{1} \\
0 \\
0
\end{array}\right], \quad \mathbf{r}_{B_{2}}^{1}=\left[\begin{array}{c}
1 \\
c_{\varepsilon_{2}} b_{2} \\
s_{\varepsilon_{2}} b_{2} \\
0
\end{array}\right], \quad \mathbf{r}_{B_{3}}^{1}=\left[\begin{array}{c}
1 \\
c_{\varepsilon_{3}} b_{3} \\
s_{\varepsilon_{3}} b_{3} \\
0
\end{array}\right] .
$$

As a consequence, the 3-RPS parallel manipulators under study are characterized by eight design parameters, namely $a_{1}, a_{2}, a_{3}, b_{1}, b_{2}, b_{3}, \varepsilon_{2}, \varepsilon_{3}$.

\subsection{Targeted application: ankle rehabilitation}

The 3-RPS parallel manipulator described in Fig. 1 will be used as an ankle rehabilitation device. The parallel manipulator should be wearable, thus the base, composed of three revolute joints, can be hooked to the patient's lower leg. The moving-platform, composed of three spherical joints, will be placed on the foot palm. This application is illustrated in Fig. 2.

The key movements of the ankle joint are plantarflexion-dorsiflexion, abduction-adduction and inversion-eversion, which occur in the sagittal, transverse and frontal planes, respectively. Combinations of these motions yield 3-dof motions. As the foot can never execute pure inversion and eversion, the ankle joint cannot perform 3-dof rotational motion. On the contrary, the foot can only perform supination and pronation, which are made up of movement in all three planes (frontal, sagittal, and transverse planes).

Plantaflexion-dorsiflexion are the dominant motions of the ankle joint which occur primarily in the sagittal plane. Several studies have indicated that the maximum required range of motion of plantaflexion-dorsiflexion for walking in everyday activities is $30^{\circ}$. Therefore, the orientation capability of the 3-RPS parallel manipulator synthesized in this paper is the utmost concern and should be larger than $30^{\circ}$.

As stated in Section 2.1, the ankle rehabilitation device depicted in Fig. 2 is characterized by eight design parameters, namely $a_{1}, a_{2}, a_{3}, b_{1}, b_{2}, b_{3}, \varepsilon_{2}, \varepsilon_{3}$. It should be noted that they are not independent and their relations depend on the constraints imposed to the moving-platform as discussed hereafter. 


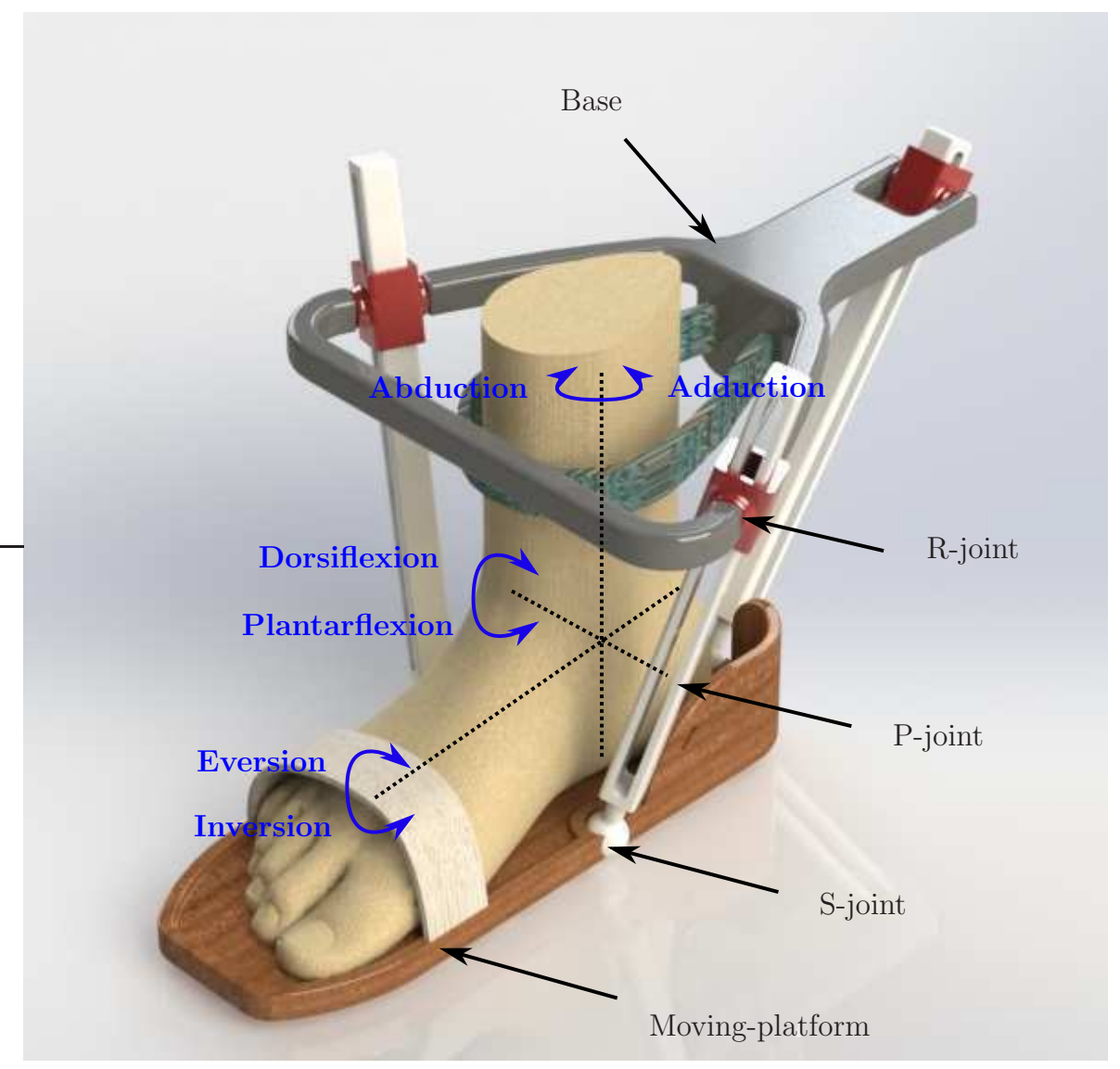

Figure 2: Application for ankle rehabilitation device 


\section{Constraint-based design conditions}

The eight design parameters $a_{1}, a_{2}, a_{3}, b_{1}, b_{2}, b_{3}, \varepsilon_{2}, \varepsilon_{3}$ are not independent to each other as they are related to each other by the constraints imposed to the manipulator [25]. Each constraint represents certain motions or operation modes. The initial step to derive the constraint-based design conditions is to carry out the coordinate transformation of point $B_{i}$ on the movingplatform by means of Euler parametrization. Once the coordinates of all points are expressed in terms of Euler parametrization, the constraint equation can be formulated by examining its geometric conditions.

The coordinates of point $B_{i}$ expressed in the fixed frame $\Sigma_{0}$ can be determined through transformation, i.e. $\mathbf{r}_{B_{i}}^{0}=\mathbf{M} \mathbf{r}_{B_{i}}^{1}$. Matrix $\mathbf{M}$ is a transformation matrix defined as follows:

$$
\mathbf{M}=\left(\begin{array}{cccc}
x_{0}^{2}+x_{1}^{2}+x_{2}^{2}+x_{3}^{2} & 0 & 0 & 0 \\
X & x_{0}^{2}+x_{1}^{2}-x_{2}^{2}-x_{3}^{2} & 2\left(x_{1} x_{2}-x_{0} x_{3}\right) & 2\left(x_{1} x_{3}+x_{0} x_{2}\right) \\
Y & 2\left(x_{1} x_{2}+x_{0} x_{3}\right) & x_{0}^{2}-x_{1}^{2}+x_{2}^{2}-x_{3}^{2} & 2\left(x_{2} x_{3}-x_{0} x_{1}\right) \\
Z & 2\left(x_{1} x_{3}-x_{0} x_{2}\right) & 2\left(x_{2} x_{3}+x_{0} x_{1}\right) & x_{0}^{2}-x_{1}^{2}-x_{2}^{2}+x_{3}^{2}
\end{array}\right)
$$

The parameters $x_{0}, x_{1}, x_{2}, x_{3}$, which appear in matrix $\mathbf{M}$, are called Euler parameters of the rotation. They are useful in the representation of a spatial Euclidean displacement.

As the coordinates of point $B_{i}$ are then expressed in the fixed frame, the constraint equation can be derived. The constraint equation describes the motion of point $B_{i}$ of spherical joint in the plane of normal vector $\mathbf{s}_{i}$ and passing through point $A_{i}$. Each pair of vertices $A_{i}$ and $B_{i}$ is connected by a prismatic joint. The prismatic length is denoted as $r_{i}$. Since the $i$-th prismatic length is orthogonal to the revolute axis $\mathbf{s}_{i}$, the scalar product of vector $\left(\mathbf{r}_{B_{i}}^{0}-\mathbf{r}_{A_{i}}^{0}\right)$ and vector $\mathbf{s}_{i}$ vanishes, namely:

$$
\left(\mathbf{r}_{B_{i}}^{0}-\mathbf{r}_{A_{i}}^{0}\right)^{T} \mathbf{s}_{i}=0, \quad i=1,2,3 .
$$

After computing the scalar product for all three legs and eliminating the common denominator, the constraint equations come out:

$$
\begin{aligned}
& g_{1}: Y+\left(2 x_{0} x_{3}+2 x_{1} x_{2}\right) b_{1}=0 \\
& g_{2}: 4 c_{\varepsilon_{2}}^{2} b_{2} x_{1} x_{2}-2\left(x_{1}^{2}-x_{2}^{2}\right) c_{\varepsilon_{2}} s_{\varepsilon_{2}} b_{2}+c_{\varepsilon_{2}} Y-s_{\varepsilon_{2}} X+\left(2 x_{0} x_{3}-2 x_{1} x_{2}\right) b_{2}=0 \\
& g_{3}: 4 c_{\varepsilon_{3}}^{2} b_{3} x_{1} x_{2}-2\left(x_{1}^{2}-x_{2}^{2}\right) c_{\varepsilon_{3}} s_{\varepsilon_{3}} b_{3}+c_{\varepsilon_{3}} Y-s_{\varepsilon_{3}} X+\left(2 x_{0} x_{3}-2 x_{1} x_{2}\right) b_{3}=0
\end{aligned}
$$

Those three constraint equations are written as polynomial ideal $\mathcal{I}=\left\langle g_{1}, g_{2}, g_{3}\right\rangle$ with variables $\left\langle x_{0}, x_{1}, x_{2}, x_{3}, X, Y, Z\right\rangle$. The primary decomposition is computed and it turns out that $\mathcal{I}$ cannot be decomposed into several sub-ideals. However, the primary decomposition returns the new formulation of ideal $\mathcal{I}$ as $\mathcal{I}=\left\langle h_{1}, h_{2}, h_{3}\right\rangle$, where $h_{1}, h_{2}, h_{3}$ take the form:

$$
\begin{aligned}
& h_{1}: \alpha_{1}\left(x_{1}^{2}+x_{2}^{2}\right)+\alpha_{2} x_{1} x_{2}+\alpha_{3} x_{0} x_{3}=0 \\
& h_{2}: \beta_{1}\left(x_{1}^{2}+x_{2}^{2}\right)+\beta_{2} x_{1} x_{2}+\beta_{3} Y=0 \\
& h_{3}: \gamma_{1}\left(x_{1}^{2}+x_{2}^{2}\right)+\gamma_{2} x_{1} x_{2}+\gamma_{3} X=0
\end{aligned}
$$

where $\alpha_{j}, \beta_{j}, \gamma_{j}$ for $j=1,2,3$ are polynomial coefficients that are functions of the eight design parameters. 
From Eq. (7), it is apparent that $h_{1}, h_{2}, h_{3}$ are free of $Z$ parameter. It means that the 3-RPS parallel manipulator under study can perform pure translational motion along $z$-axis for any value of the design parameters. It should be noted that $h_{2}$ and $h_{3}$ are linearly dependent on the parameters $X$ and $Y$. It means that the manipulator undergoes translational motions along $x$ and $y$ axes which are coupled to rotations. Those translational motions are named parasitic motions [28, 29, 30].

In the following, the orientation parameters $x_{0}, x_{1}, x_{2}, x_{3}$ from $h_{1}, h_{2}, h_{3}$ are constrained to be null successively or in pairs, which leads to different operation modes and motion types. By fulfilling these conditions, several design conditions can be mathematically formulated to generate new architectures of 3-RPS parallel manipulator.

\subsection{Design Condition 1}

From Eq. (7), we can notice that only $h_{1}$ is in a function of both parameters $x_{0}$ and $x_{3}$. When either $x_{0}$ or $x_{3}$ vanishes, equation $h_{1}$ becomes:

$$
h_{1}: \alpha_{1}\left(x_{1}^{2}+x_{2}^{2}\right)+\alpha_{2} x_{1} x_{2}=0
$$

It means that the following derivation of design conditions applies to both constraints $x_{0}=0$ and $x_{3}=0$.

All polynomial coefficients $\alpha_{1}, \alpha_{2}$ have to vanish and one has to discuss the new ideal $\mathcal{J}=$ $\left\langle\alpha_{1}, \alpha_{2}\right\rangle$. The Groebner basis with lexicographic order is computed for ideal $\mathcal{J}$ and 17 solutions are obtained. Not all solutions are possible and hence some assumptions are formulated, as follows:

1. The second and the third legs cannot be coincident with the first leg: $\varepsilon_{2} \neq 0^{\circ}$ and $\varepsilon_{3} \neq 0^{\circ}$

2. The second leg cannot be coincident with the third leg: $\varepsilon_{2} \neq \varepsilon_{3}$ or $b_{2} \neq b_{3} \neq 0$

3. $b_{i}(i=1,2,3)$ should be positive: $b_{i} \geq 0, i=1,2,3$

4. The moving-platform cannot be a point: $b_{1} \neq b_{2} \neq b_{3} \neq 0$

5. There is no complex solution: $\left\{b_{1}, b_{2}, b_{3}, \varepsilon_{2}, \varepsilon_{3}\right\} \in \mathbb{R}$

After removing the solutions that do not fulfil the foregoing assumptions, the following three design conditions are obtained:

$$
\begin{aligned}
& 1 \mathrm{~A}: a_{2}=\frac{a_{1}}{t_{\varepsilon_{3}}}, a_{3}=0, b_{2}=\frac{b_{1}}{t_{\varepsilon_{3}}}, b_{3}=0, \varepsilon_{2}=\frac{\pi}{2}, \varepsilon_{3} \neq 0 \text { or } \varepsilon_{3} \neq \pm \pi \\
& 1 \mathrm{~B}: a_{2}=-\frac{a_{1}}{t_{\varepsilon_{3}}}, a_{3}=0, b_{2}=-\frac{b_{1}}{t_{\varepsilon_{3}}}, b_{3}=0, \varepsilon_{2}=-\frac{\pi}{2}, \varepsilon_{3} \neq 0 \text { or } \varepsilon_{3} \neq \pm \pi \\
& 1 \mathrm{C}: a_{1}=a_{3} \frac{c_{\left(\varepsilon_{2}-\varepsilon_{3}\right)}}{c_{\varepsilon_{2}}}, a_{2}=a_{3} \frac{c_{\varepsilon_{3}}}{c_{\varepsilon_{2}}}, b_{1}=b_{3} \frac{c_{\left(\varepsilon_{2}-\varepsilon_{3}\right)}}{c_{\varepsilon_{2}}}, b_{2}=b_{3} \frac{c_{\varepsilon_{3}}}{c_{\varepsilon_{2}}}, \varepsilon_{2} \neq \pm \frac{\pi}{2} \text { or } \varepsilon_{2} \neq \pm \frac{3 \pi}{2}
\end{aligned}
$$

The 3-RPS parallel manipulator can be generated by selecting one of the three design conditions. Accordingly, the obtained manipulator will have two operation modes, namely 
$x_{0}=0$ and $x_{3}=0$. All feasible poses of the 3-RPS parallel manipulator in operation mode $x_{0}=0$ are determined from the home configuration by making a $\pi$-rotation angle about an axis combined with a translational motion along the same axis. In the second operation mode $x_{3}=0$, the poses are obtained by rotating and translating about a transformation axis of which the direction is parallel to the $x y$-plane. These two operation modes have been discussed thoroughly in [7].

\subsection{Design Condition 2}

It can be seen from Eq. (7) that $h_{1}, h_{2}, h_{3}$ are in terms of parameters $x_{1}$ and $x_{2}$. When parameter $x_{1}$ vanishes, equations $h_{1}, h_{2}, h_{3}$ yields:

$$
\begin{aligned}
& h_{1}: \alpha_{1} x_{2}^{2}+\alpha_{3} x_{0} x_{3}=0 \\
& h_{2}: \beta_{1} x_{2}^{2}+\beta_{3} Y=0 \\
& h_{3}: \gamma_{1} x_{2}^{2}+\gamma_{3} X=0
\end{aligned}
$$

If parameter $x_{2}$ vanishes, equations $h_{1}, h_{2}, h_{3}$ will have the same polynomial coefficients as in Eq. (10). It means that the following derivation of design conditions applies to both constraints $x_{1}=0$ and $x_{2}=0$.

All polynomial coefficients $\alpha_{1}, \alpha_{3}, \beta_{1}, \beta_{3}, \gamma_{1}, \gamma_{3}$ should vanish and are written as polynomial ideal $\mathcal{J}=\left\langle\alpha_{1}, \alpha_{3}, \beta_{1}, \beta_{3}, \gamma_{1}, \gamma_{3}\right\rangle$. The Groebner basis with lexicographic order is computed over the ideal $\mathcal{J}$ and leads to 11 solutions. Not all solutions are feasible and only solutions fulfilling aforementioned assumptions are kept. Thus, three design conditions are obtained as follows:

$$
\begin{aligned}
2 \mathrm{~A}: a_{2} & =-\frac{a_{1}}{t_{\varepsilon_{3}}}, a_{3}=0, \varepsilon_{2}=\frac{\pi}{2}, b_{2}=-\frac{b_{1}}{t_{\varepsilon_{3}}}, b_{3}=0, \varepsilon_{2}=\frac{\pi}{2}, \varepsilon_{3} \neq 0 \text { or } \\
\varepsilon_{3} & \neq \pm \pi \\
2 \mathrm{~B}: a_{2} & =\frac{a_{1}}{t_{\varepsilon_{3}}}, a_{3}=0, b_{2}=\frac{b_{1}}{t_{\varepsilon_{3}}}, b_{3}=0, \varepsilon_{2}=-\frac{\pi}{2}, \varepsilon_{3} \neq 0 \text { or } \varepsilon_{3} \neq \pm \pi \\
2 \mathrm{C}: a_{1} & =-a_{3} \frac{c_{\left(\varepsilon_{2}-\varepsilon_{3}\right)}}{c_{\varepsilon_{2}}}, a_{2}=a_{3} \frac{c_{\varepsilon_{3}}}{c_{\varepsilon_{2}}}, b_{1}=-b_{3} \frac{c_{\left(\varepsilon_{2}-\varepsilon_{3}\right)}}{c_{\varepsilon_{2}}}, b_{2}=b_{3} \frac{c_{\varepsilon_{3}}}{c_{\varepsilon_{2}}}, \varepsilon_{2} \neq \pm \frac{\pi}{2} \text { or } \varepsilon_{2} \neq \pm \frac{3 \pi}{2}
\end{aligned}
$$

By selecting one of three design conditions, the 3-RPS parallel manipulator can be generated. This manipulator will have two operation modes, namely $x_{1}=0$ and $x_{2}=0$. The 3-RPS parallel manipulators with operation modes $x_{1}=0$ and $x_{2}=0$ have not been discussed before and will be analysed in more details in this paper. Let us assume that none of the design parameters can be null, thus the only possible design condition is $2 \mathrm{C}$. This design condition is applied to generate the 3-RPS parallel manipulator for ankle rehabilitation device as shown in Fig. 2.

\subsection{Constraint equations of design condition 2C for ankle rehabili- tation device}

The architecture of ankle rehabilitation device shown in Fig. 2 is generated by selecting the design condition $2 \mathrm{C}$. By substituting the design condition $2 \mathrm{C}$ into the constraint equations 
described in Eq. (6), the number of unknowns can be reduced from 8 to 4 , namely: $a_{3}, b_{3}, \varepsilon_{2}, \varepsilon_{3}$. The constraint equations take the form:

$$
\begin{aligned}
& \tilde{g}_{1}: c_{\varepsilon_{2}} Y-2 c_{\left(\varepsilon_{2}-\varepsilon_{3}\right)}\left(x_{0} x_{3}+x_{1} x_{2}\right) b_{3}=0 \\
& \left.\tilde{g}_{2}:-s_{\varepsilon_{2}} c_{\varepsilon_{2}} X+c_{\varepsilon_{2}}^{2} Y-2 c_{\varepsilon_{3}} s_{\varepsilon_{2}} c_{\varepsilon_{2}} x_{1}^{2}-s_{\varepsilon_{2}} c_{\varepsilon_{2}} x_{2}^{2}-2 c_{\varepsilon_{2}}^{2} x_{1} x_{2}-x_{0} x_{3}+x_{1} x_{2}\right) b_{3}=0 \\
& \tilde{g}_{3}:-s_{\varepsilon_{3}} X+c_{\varepsilon_{3}} Y+\left(-2 s_{\varepsilon_{3}} c_{\varepsilon_{3}} x_{1}^{2}+2 s_{\varepsilon_{3}} c_{\varepsilon_{3}} x_{2}^{2}+4 x_{1} x_{2} c_{\varepsilon_{3}}^{2}+2 x_{0} x_{3}-2 x_{1} x_{2}\right) b_{3}=0
\end{aligned}
$$

Other three constraint equations corresponding to the actuation scheme or inverse kinematics are derived. The prismatic joint in each leg is assumed to be actuated, hence the distance between points $A_{i}$ and $B_{i}$ is denoted as $r_{i}$. Once the $i$-th prismatic joint is locked, point $B_{i}$ is free to move along a circle of center $A_{i}$ and of radius $r_{i}$. This motion can be mathematically formulated as follows: $\left\|\mathbf{r}_{B_{i}}^{0}-\mathbf{r}_{A_{i}}^{0}\right\|^{2}-r_{i}^{2}=0, i=1,2,3$. It leads to the following three constraint equations:

$$
\begin{aligned}
& \tilde{g}_{4}: c_{\varepsilon_{2}}^{2} X^{2}+2 c_{\left(\varepsilon_{2}-\varepsilon_{3}\right)} c_{\varepsilon_{2}} X a_{3}-2 c_{\left(\varepsilon_{2}-\varepsilon_{3}\right)} c_{\varepsilon_{2}}\left(x_{0}^{2}+x_{1}^{2}-x_{2}^{2}-x_{3}^{2}\right) X b_{3}+c_{\varepsilon_{2}}^{2} Y^{2} \ldots=0 \\
& \tilde{g}_{5}: c_{\varepsilon_{2}}^{2} X^{2}-2 c_{\varepsilon_{3}} c_{\varepsilon_{2}}^{2} X a_{3}-2 c_{\varepsilon_{3}} c_{\varepsilon_{2}}\left(2 x_{0} x_{3} s_{\varepsilon_{2}}-2 x_{1} x_{2} s_{\varepsilon_{2}}-c_{\varepsilon_{2}} x_{0}^{2}-c_{\varepsilon_{2}} x_{1}^{2}+c_{\varepsilon_{2}} \ldots=0\right. \\
& \tilde{g}_{6}: X^{2}-2 c_{\varepsilon_{3}} X a_{3}+\left(-4 s_{\varepsilon_{3}} x_{0} x_{3}+4 s_{\varepsilon_{3}} x_{1} x_{2}+2 c_{\varepsilon_{3}} x_{0}^{2}+2 x_{1}^{2} c_{\varepsilon_{3}}-2 c_{\varepsilon_{3}} x_{2}^{2}-2 \ldots=0\right.
\end{aligned}
$$

As the expressions of $\tilde{g}_{4}, \tilde{g}_{5}, \tilde{g}_{6}$ are very lengthy, only the first line of each equation is written in Eq. (13).

The rotational parameters $x_{0}, x_{1}, x_{2}, x_{3}$ should satisfy the normalization equation based on [3] and it is defined as the seventh constraint equation:

$$
\tilde{g}_{7}: x_{0}^{2}+x_{1}^{2}+x_{2}^{2}+x_{3}^{2}-1=0
$$

The set of constraint equations is defined as a new ideal $\mathcal{K}=\left\langle\tilde{g}_{1}, \tilde{g}_{2}, \tilde{g}_{3}, \tilde{g}_{4}, \tilde{g}_{5}, \tilde{g}_{6}, \tilde{g}_{7}\right\rangle$. The computation of Hilbert dimension of ideal $\mathcal{K}$ is carried out in both operation modes $x_{1}=0$ and $x_{2}=0$. By treating the joint variable $r_{i}$ as unknown, it turns out that:

$$
\begin{aligned}
& \operatorname{dim}\left(\mathcal{K}_{x_{1}=0}\right)=3 \\
& \operatorname{dim}\left(\mathcal{K}_{x_{2}=0}\right)=3
\end{aligned}
$$

where dim denotes the dimension over $\mathbb{R}\left[a_{3}, b_{3}, \varepsilon_{2}, \varepsilon_{3}\right]$. Equations (15a)-(15b) shows that the operation modes $x_{1}=0$ and $x_{2}=0$ amount to 3 -dof motions. In the following, the operation modes $x_{1}=0$ and $x_{2}=0$ will be parametrized and physically interpreted through its orientation workspace.

\section{Orientation workspace and singularity analysis}

\subsection{Orientation workspace}

The moving-platform in both operation modes can perform coupled rotational motions and a vertical translational motion. However, the rotational motions in each operation mode cannot be easily distinguished. Therefore, the manipulator workspace associated with each operation 
mode is parametrized to recognize the physical characteristics of position and orientation of the moving platform.

From Sec. 3, the position and orientation of the moving-platform are characterized by parameters $x_{0}, x_{1}, x_{2}, x_{3}, X, Y, Z$. Parameters $X, Y, Z$ are the Cartesian coordinates of point $Q$ (the origin of moving-platform frame) expressed in the fixed frame $\Sigma_{0}$. For a better understanding of the orientation capability of the manipulator at hand, the Euler parameters $x_{0}, x_{1}, x_{2}, x_{3}$ are expressed in terms of azimuth $\phi \in\left[-180^{\circ}, 180^{\circ}\right]$ and tilt $\theta \in\left[0,180^{\circ}\right][31,32]$ as follows:

$$
\begin{aligned}
x_{0} & =\cos \left(\frac{\theta}{2}\right) \cos \left(\frac{\phi}{2}+\frac{\psi}{2}\right) & x_{2} & =\sin \left(\frac{\theta}{2}\right) \sin \left(\frac{\phi}{2}-\frac{\psi}{2}\right) \\
x_{1} & =\sin \left(\frac{\theta}{2}\right) \cos \left(\frac{\phi}{2}-\frac{\psi}{2}\right) & x_{3} & =\cos \left(\frac{\theta}{2}\right) \sin \left(\frac{\phi}{2}+\frac{\psi}{2}\right)
\end{aligned}
$$

As parameter $x_{1}$ is null in the first operation mode $x_{1}=0$, the torsion angle can be parametrized, i.e. $\psi=\phi-\pi$. The remaining orientation parameters are converted into the tilt and azimuth angles, i.e. $x_{0}=\cos \left(\frac{\theta}{2}\right) \sin (\phi), x_{2}=\sin \left(\frac{\theta}{2}\right), x_{3}=-\cos \left(\frac{\theta}{2}\right) \cos (\phi)$. The rotation matrix in the first operation mode is denoted by $\mathbf{R}_{x_{1}=0}$, that takes form:

$$
\mathbf{R}_{x_{1}=0}=\left(\begin{array}{ccc}
-2 c_{\frac{\theta}{2}}^{2} c_{\phi}^{2}+2 c_{\frac{\theta}{2}}^{2}-1 & 2 c_{\frac{\theta}{2}}^{2} s_{\phi} c_{\phi} & 2 c_{\frac{\theta}{2}}^{2} s_{\phi} s_{\frac{\theta}{2}} \\
-2 c_{\frac{\theta}{2}}^{2} s_{\phi} c_{\phi} & -2 c_{\frac{\theta}{2}}^{2} c_{\phi}^{2}+1 & -2 s_{\frac{\theta}{2}} c_{\frac{\theta}{2}} c_{\phi} \\
-2 c_{\frac{\theta}{2}} s_{\frac{\theta}{2}} s_{\phi} & -2 s_{\frac{\theta}{2}} c_{\frac{\theta}{2}} c_{\phi} & 2 c_{\frac{\theta}{2}}^{2}-1
\end{array}\right)
$$

where $c_{\frac{\theta}{2}}=\cos \left(\frac{\theta}{2}\right), s_{\frac{\theta}{2}}=\sin \left(\frac{\theta}{2}\right), s_{\phi}=\sin (\phi)$, and $c_{\phi}=\cos (\phi)$.

In the second operation mode, the parameter $x_{2}$ is null and the torsion angle can be parametrized, i.e. $\psi=\phi$. The remaining orientation parameters are converted into the tilt and azimuth angles, i.e. $x_{0}=\cos \left(\frac{\theta}{2}\right) \cos (\phi), x_{1}=\sin \left(\frac{\theta}{2}\right)$, and $x_{3}=\cos \left(\frac{\theta}{2}\right) \sin (\phi)$. The rotation matrix in the second operation mode is denoted by $\mathbf{R}_{x_{2}=0}$, that takes form:

$$
\mathbf{R}_{x_{2}=0}=\left(\begin{array}{ccc}
2 c_{\frac{\theta}{2}}^{2} c_{\phi}^{2}-2 c_{\frac{\theta}{2}}^{2}+1 & -2 c_{\frac{\theta}{2}}^{2} s_{\phi} c_{\phi} & 2 s_{\frac{\theta}{2}} c_{\frac{\theta}{2}} s_{\phi} \\
2 c_{\frac{\theta}{2}}^{2} s_{\phi} c_{\phi} & 2 c_{\frac{\theta}{2}}^{2} c_{\phi}^{2}-1 & -2 c_{\frac{\theta}{2}} s_{\frac{\theta}{2}} c_{\phi} \\
2 c_{\frac{\theta}{2}} s_{\frac{\theta}{2}} s_{\phi} & 2 s_{\frac{\theta}{2}} c_{\frac{\theta}{2}} c_{\phi} & 2 c_{\frac{\theta}{2}}^{2}-1
\end{array}\right)
$$

Without loss of generality, let us consider the 3-RPS parallel manipulator with the following design parameter values: $a_{3}=1, b_{3}=0.5, \varepsilon_{2}=66^{\circ}, \varepsilon_{3}=-66^{\circ}$. The vertical translational motion of the moving-platform can be easily grasped. However, it is more difficult to distinguish its 2-dof rotational motions. The moving-platform is assumed to work at given height $Z=1$. Hence, the 2-dof motion performed by this manipulator in both operation modes $x_{1}=0$ and $x_{2}=0$ are characterized by the rotational matrices defined in Eqs. (17)-(18).

Let us consider the geometric center $P$ of the moving-platform denoted by $P$ of coordinates

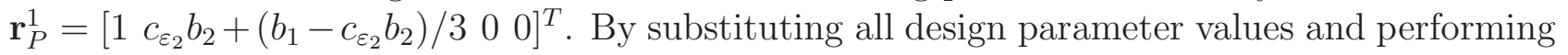
coordinate transformation via rotation matrices defined in Eqs. (17)-(18), the coordinates of point $P$ can be obtained in the fixed frame $\left(\Sigma_{0}\right)$ in both operation modes.

The 2-dof rotational motions are then represented by the point reachable workspace generated by the geometric center of moving-platform, point $P$. It reveals that the 2 -dof rotational 


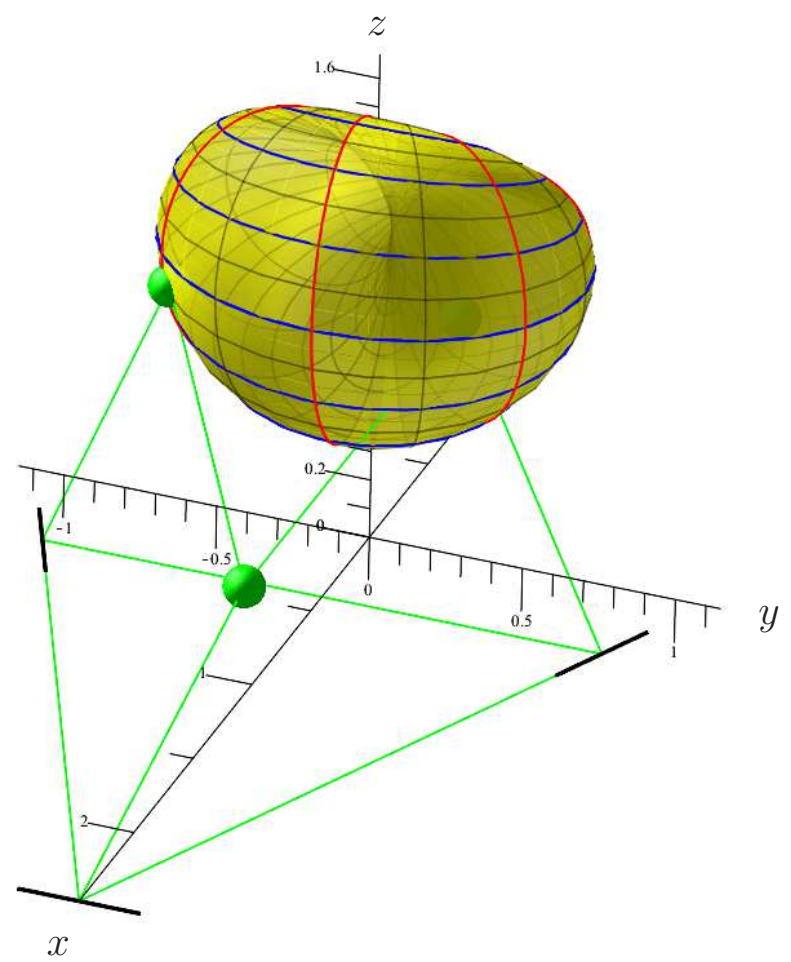

Figure 3: A manipulator pose in operation mode $x_{1}=0$

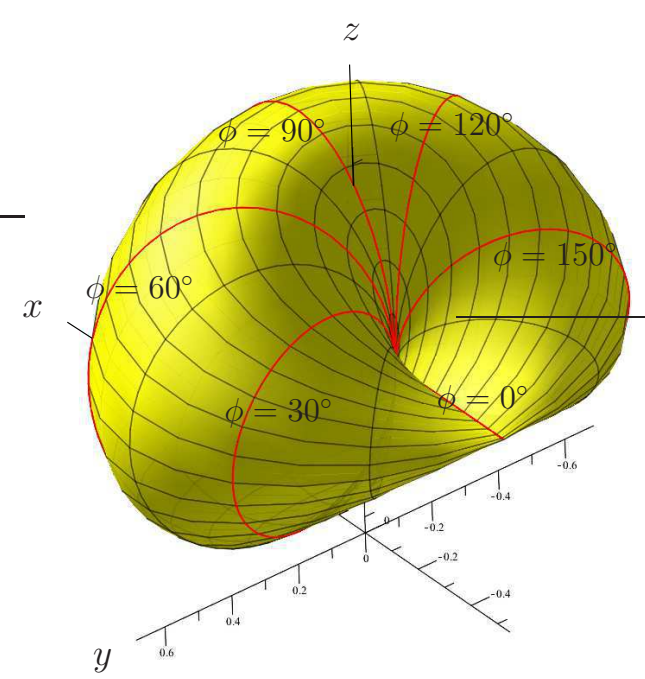

(a) 1-dof motion by $\phi$

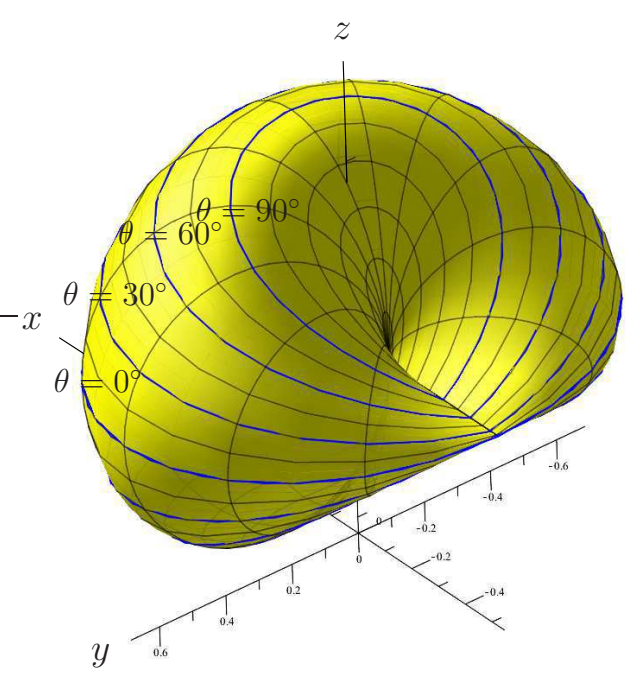

(b) 1-dof motion by $\theta$

Figure 4: Rotational motion presented by $\phi$ and $\theta$ in operation mode $x_{1}=0$. 
motions in both operation modes are bounded by a particular surface, called the Cross Cap surface, in both operation modes. This surface has a shape of shrunk Torus-like where the side is clipped so that they cross, as shown in Figs. 3 and 5 for operation modes $x_{1}=0$ and $x_{2}=0$, respectively.

Given any value of tilt angle $\theta$, the geometric center of moving-platform $P$ can follow the red curves as shown in Figs. 4(a) and 6(a) for operation modes $x_{1}=0$ and $x_{2}=0$, respectively. When the azimuth angle is null $\left(\phi=0^{\circ}\right)$, the trajectory becomes a straight line. When the azimuth angle is increased, the trajectory is no longer a straight line but an ellipse. Starting from $\phi=30^{\circ}$, the ellipses move clockwise until they comeback to $\phi=0^{\circ}$.

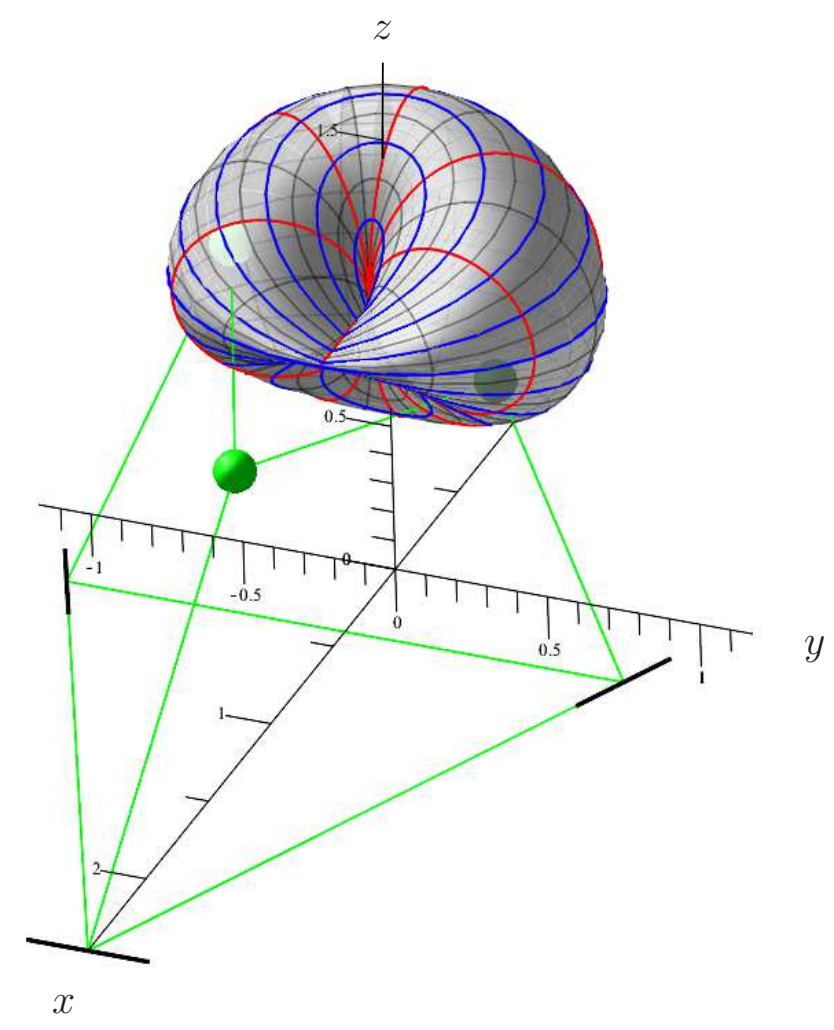

Figure 5: A manipulator pose in operation mode $x_{2}=0$

On the other hand, for any given value of azimuth angle $\phi$, the geometric center of movingplatform follows the blue curves as depicted in Figs. 4(b) and 6(b) for operation modes $x_{1}=0$ and $x_{2}=0$, respectively. When the tilt angle is null $\left(\theta=0^{\circ}\right)$, the trajectory becomes an ellipse, which is the equator of the Cross Cap surface. When the tilt angle is increased, the trajectory changes symmetrically with respect to its equator. Eventually, when the tilt angle reaches $\theta=180^{\circ}$, the trajectory becomes a point, located at the center of the Cross Cap surface.

The point reachable workspace of 2-dof rotational motions generated by the geometric center $P$ in both operation modes, are bounded by Cross Cap surfaces. They behave similarly over the angles $\phi$ and $\theta$. However, the Cross Cap surfaces associated to both operation modes have different orientations and they are facing toward different directions. The clipped side of the Cross Cap surface belonging to operation mode $x_{1}=0$ faces toward negative $x$-axis, while 


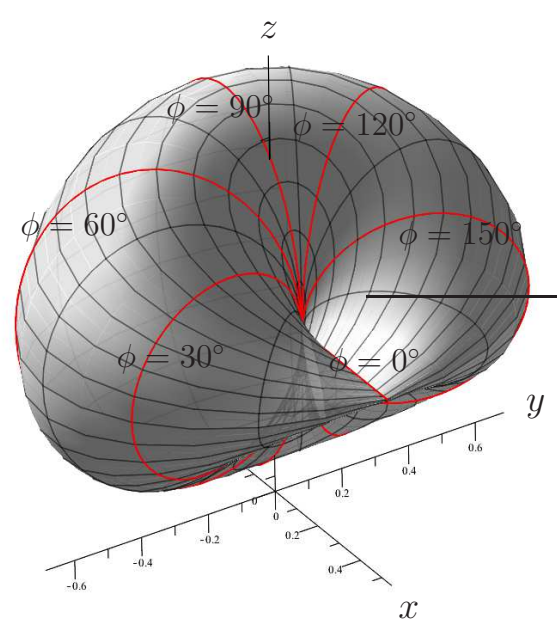

(a) 1-dof motion by $\phi$

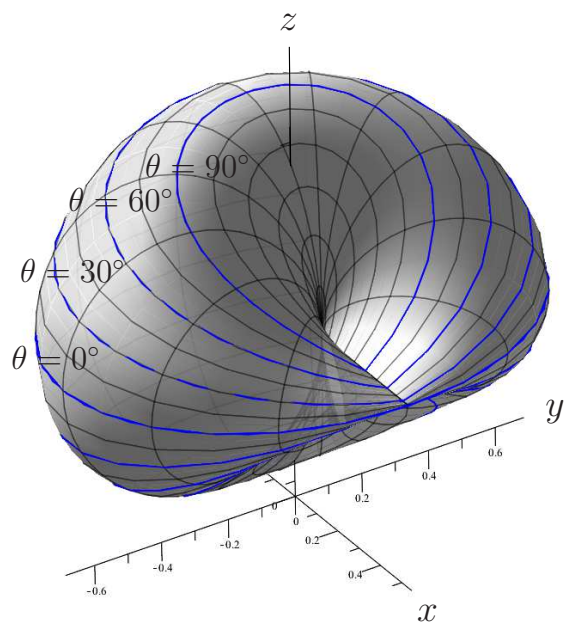

(b) 1-dof motion by $\theta$

Figure 6: Rotational motion presented by $\phi$ and $\theta$ in operation mode $x_{2}=0$.

the one belonging to operation mode $x_{2}=0$ faces toward positive $x$-axis. The combination of both workspaces is shown in Fig. 7.

The trajectories of point $P$ for variations of azimuth $\phi \in\left[-180^{\circ}, 180^{\circ}\right]$ and tilt $\theta \in\left[0^{\circ}, 180^{\circ}\right]$ are not similarly reflected over the entire workspace. It emphasizes that the orientation workspace of the 3-RPS parallel manipulator under study is not symmetrical.

\subsection{Singularity analysis}

A parallel manipulator reaches a parallel singularity when the determinant of its forward Jacobian matrix vanishes. The forward Jacobian of the 3-RPS parallel manipulator is obtained upon differentiation of the seven constraint equations $\left(\tilde{g}_{1}, \tilde{g}_{2}, \tilde{g}_{3}, \tilde{g}_{4}, \tilde{g}_{5}, \tilde{g}_{6}, \tilde{g}_{7}\right)$ expressed in Eqs. (12)(14), with respect to the seven parameters $x_{0}, x_{1}, x_{2}, x_{3}, X, Y, Z$, as follows:

$$
\mathbf{J}=\left(\frac{\partial \tilde{g}_{j}}{\partial x_{0}}, \frac{\partial \tilde{g}_{j}}{\partial x_{1}}, \frac{\partial \tilde{g}_{j}}{\partial x_{2}}, \frac{\partial \tilde{g}_{j}}{\partial x_{3}}, \frac{\partial \tilde{g}_{j}}{\partial X}, \frac{\partial \tilde{g}_{j}}{\partial Y}, \frac{\partial \tilde{g}_{j}}{\partial Z}\right), \quad j=1, \ldots, 7
$$

Then the determinant of the forward Jacobian matrix is computed in both operation modes $x_{1}=0$ and $x_{2}=0$. It appears that this determinant splits into two factors in both operation modes:

$$
\begin{aligned}
& \operatorname{det}\left(\mathbf{J}_{x_{1}=0}\right)=x_{2} \cdot f_{1}\left(a_{3}, b_{3}, \varepsilon_{2}, \varepsilon_{3}, x_{0}, x_{2}, x_{3}, Z\right)=0 \\
& \operatorname{det}\left(\mathbf{J}_{x_{2}=0}\right)=x_{1} \cdot f_{2}\left(a_{3}, b_{3}, \varepsilon_{2}, \varepsilon_{3}, x_{0}, x_{1}, x_{3}, Z\right)=0
\end{aligned}
$$

The two factors correspond to two types of parallel singularities, namely constraint and actuation singularities [33, 34]. These singularities will be further investigated hereafter.

\subsubsection{Constraint singularity}

The first factors in Eqs. (20)-(21) lead to the constraint singularity configurations that belong to both operation modes simultaneously, i.e. $x_{1}=x_{2}=0$. By substituting the orientation 


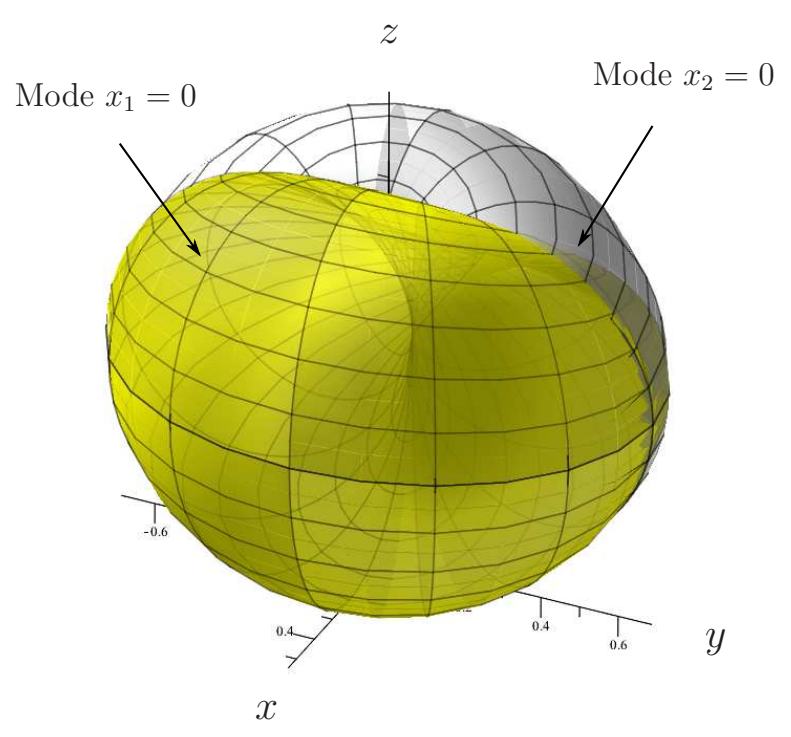

Figure 7: Combination of Cross Cap surfaces

parametrization defined in Eq. (16) into Eqs. (20)-(21), the constraint singularity condition can be derived. A constraint singularity occurs if and only if the tilt angle is null for any given azimuth angle $\phi$, hence:

$$
\text { Constraint singularity }=\left\{\theta=0^{\circ}, \forall \phi \in\left[-180^{\circ}, 180^{\circ}\right]\right\}
$$

It means that the 3-RPS parallel manipulator reaches a constraint singularity configuration when its moving-platform is parallel to the base. It should be noted that constraint singularities correspond to the transition configurations that allow the 3-RPS parallel manipulator to switch from operation mode $x_{1}=0$ to operation mode $x_{2}=0$ and vice versa.

\subsection{Actuation singularity}

The second factors in Eqs. (20)-(21) give the actuation singularity conditions inside the workspace for both operation modes $x_{1}=0$ and $x_{2}=0$, respectively. These singularities have a great impact onto the manipulator kinematic performance and the orientation capability of its movingplatform. In what follows, only actuation singularities are considered for dimension optimisation and they are defined as follows:

$$
\begin{aligned}
& \mathcal{S}_{1}: f_{1}\left(a_{3}, b_{3}, \varepsilon_{2}, \varepsilon_{3}, x_{0}, x_{2}, x_{3}, Z\right)=0 \\
& \mathcal{S}_{2}: f_{2}\left(a_{3}, b_{3}, \varepsilon_{2}, \varepsilon_{3}, x_{0}, x_{1}, x_{3}, Z\right)=0
\end{aligned}
$$

The orientation parametrizations defined in Eq. (16) are substituted into Eqs. (23)-(24) to derive the condition of actuation singularity. Without loss of generality, we consider the design of 3-RPS parallel manipulator employed in Sec. 4.1, namely $a_{3}=1, b_{3}=0.5, \varepsilon_{2}=66^{\circ}, \varepsilon_{3}=-66^{\circ}$ in operation mode $x_{2}=0$. The actuation singularity loci of the 3 -RPS parallel manipulator 
that works at given height $Z=1$ is depicted by the red curve in Polar coordinate as shown in Fig. 8(a).

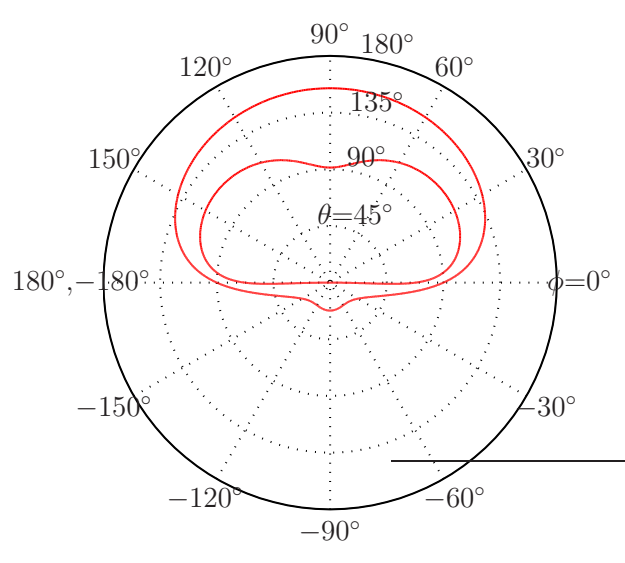

(a) In Polar coordinates

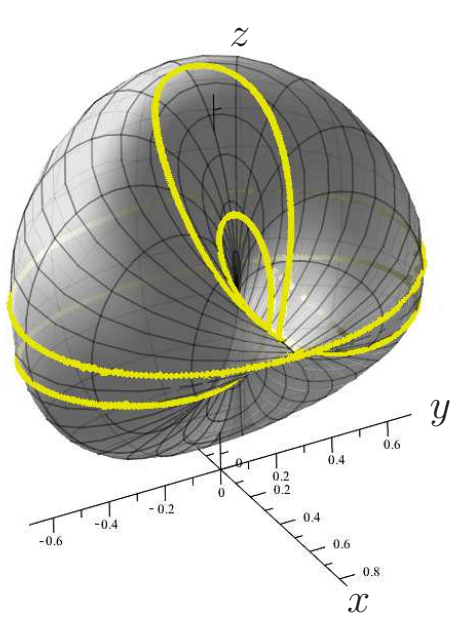

(b) In Cartesian coordinates

Figure 8: Actuation singularity loci

Let us recall the workspace of point $P$ (the geometric center of moving-platform) which is bounded by the Cross Cap surface as shown in Fig. 3. Accordingly, its singularity loci can be plotted together with the Cross Cap surface in Cartesian space as shown in Fig. 8(b). It is apparent that the workspace of the manipulator limited by the singularity loci is not symmetrical with respect to the home configuration defined by $\phi=0^{\circ}$ and $\theta=0^{\circ}$. Figures $8(\mathrm{a})$ and 8(b) show that the workspace outside the singularity loci between $\phi \in\left[-180^{\circ}, 0^{\circ}\right]$ is extremely large, however this workspace cannot be reached since the platform should pass through the singularity two times from the home pose $\left(\phi=0^{\circ}, \theta=0^{\circ}\right)$. The workspace inside the singularity loci between $\phi \in\left[0^{\circ}, 180^{\circ}\right]$ is more significant and will be considered hereafter to synthesize the design parameters $a_{3}, b_{3}, \varepsilon_{2}, \varepsilon_{3}$.

\section{Dimension Optimization}

It is well-known that the manipulator performance is strongly affected by its geometrical design parameters $a_{3}, b_{3}, \varepsilon_{2}, \varepsilon_{3}$. According to the analysis performed in Sec. 4.2, the workspace inside the singularity loci between $\phi \in\left[0^{\circ}, 180^{\circ}\right]$ is more significant and will be considered for the dimension synthesis. Here, dimension optimization aims to determine the values of design parameters $a_{3}, b_{3}, \varepsilon_{2}, \varepsilon_{3}$ by considering the maximum tilt angle $\theta$ from the home pose $\phi=$ $0^{\circ}, \theta=0^{\circ}$ without reaching actuation singularity for azimuth angle $\phi \in\left[0^{\circ}, 180^{\circ}\right]$ at given height $Z$. The design parameters in turn affect the base and moving-platform shapes and sizes.

\subsection{Boundary of Design Parameters}

The relations among eight design parameters $a_{1}, a_{2}, a_{3}, b_{1}, b_{2}, b_{3}, \varepsilon_{2}, \varepsilon_{3}$ for 3 -RPS parallel manipulator are given by design condition $2 \mathrm{C}$ in Eq. (11). This design condition reduces the number 
of unknowns from 8 to 4 , namely $a_{3}, b_{3}, \varepsilon_{2}, \varepsilon_{3}$.

However, the relation between the base and moving-platform has not been specified yet. The fact that all dimensions can be scaled up to each other, we assign the design parameter $a_{3}=1$. Design parameters $b_{3}, \varepsilon_{2}, \varepsilon_{3}$ become the remaining unknowns and should be positive. Here, $b_{3}$ is supposed to be smaller than $a_{3}$. Thus, $b_{3}$ is bounded between 0 and 1 .

As $\varepsilon_{1}$ is assigned to be $0^{\circ}$, the dimension synthesis process aims to determine the values of $\varepsilon_{2}$ and $\varepsilon_{3}$. To always fulfil the design condition $2 \mathrm{C}$ in Eq. (11), the lower and upper bounds of design parameters $\varepsilon_{2}$ and $\varepsilon_{3}$ are $\varepsilon_{2} \in\left[0^{\circ}, 90^{\circ}\right)$ and $\varepsilon_{3} \in\left[0^{\circ},-90^{\circ}\right)$, respectively. The summary of the design parameter boundaries is presented in Table 1.

Table 1: Lower and upper bounds of design parameters $b_{3}, \varepsilon_{2}$ and $\varepsilon_{3}$

\begin{tabular}{cccc}
\hline \hline & $\varepsilon_{2}$ & $\varepsilon_{3}$ & $b_{3}$ \\
\hline $\min$ & $0^{\circ}$ & $0^{\circ}$ & 0 \\
$\max$ & $90^{\circ}$ & $-90^{\circ}$ & 1 \\
\hline \hline
\end{tabular}

In what remains, the symmetrical designs are considered since they are usually simpler than their non-symmetrical counterparts $[35,36]$. Thus the relation between $\varepsilon_{2}$ and $\varepsilon_{3}$ is the following:

$$
\varepsilon_{3}=-\varepsilon_{2}
$$

As all design parameters are now given in terms of parameters $b_{3}$ and $\varepsilon_{2}$, it is possible to represent the manipulator performances into the two-dimension design space $\left(b_{3}, \varepsilon_{2}\right)$.

\subsection{Maximum Inscribed Circle Diameter (MICD)}

The design parameters $b_{3}, \varepsilon_{2}$ are determined by considering the maximum tilt angle $\theta$ from the home pose $\left(\phi=0^{\circ}, \theta=0^{\circ}\right)$ without reaching actuation singularity for azimuth angle $\phi \in\left(0,180^{\circ}\right]$ at given height $Z$.

To deal with the non-symmetrical orientation workspace, Maximum Inscribed Circle Diameter (MICD) is used. A circle is drawn until it becomes tangent to the actuation singularity loci defined by $\mathcal{S}_{1}$ and $\mathcal{S}_{2}$ (see Eqs. (23) and (24)). The corresponding tilt angle amounts to the maximum tilting capability of the manipulator, as shown in Fig. 9. The tilt angle is obtained as MICD which can be mathematically expressed as follows:

$$
\operatorname{MICD}=\max _{0^{\circ} \leq \theta \leq 180^{\circ}}\left\{\theta, \forall \phi \in\left[0^{\circ}, 180^{\circ}\right], \operatorname{det}(\mathbf{J}) \neq 0\right\}
$$

\subsection{Dimension Synthesis}

The MICD defined in Eq. (26) is employed as a basis to compare the maximum tilting capability of the 3-RPS parallel manipulator over its entire orientation workspace for a specific range of design parameters. The distributions of MICD is plotted in two-dimensional design space $\left(b_{3}, \varepsilon_{2}\right)$ explained in Sec. 5.1. 
Table 2: Distributions of MICD for given height $Z$

\begin{tabular}{llll}
\hline \hline$Z$ & Mode $x_{1}=0\left(\mathrm{~m}_{1}\right)$ & Mode $x_{2}=0\left(\mathrm{~m}_{2}\right)$ & Optimum region \\
\hline
\end{tabular}

1

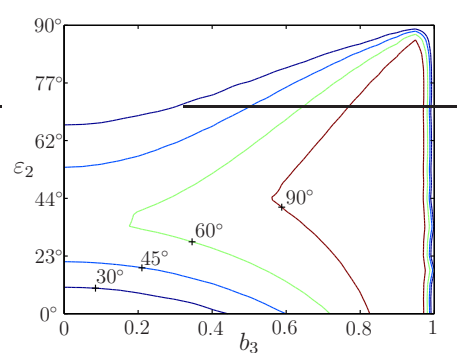

1.25

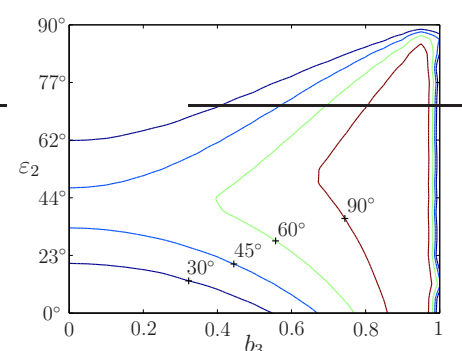

1.5

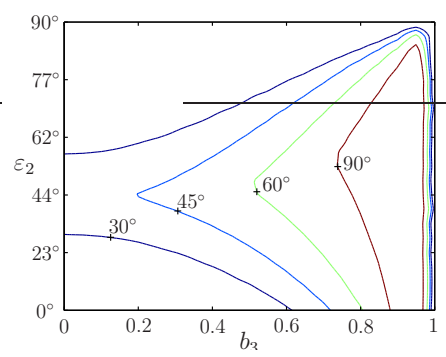

1.75
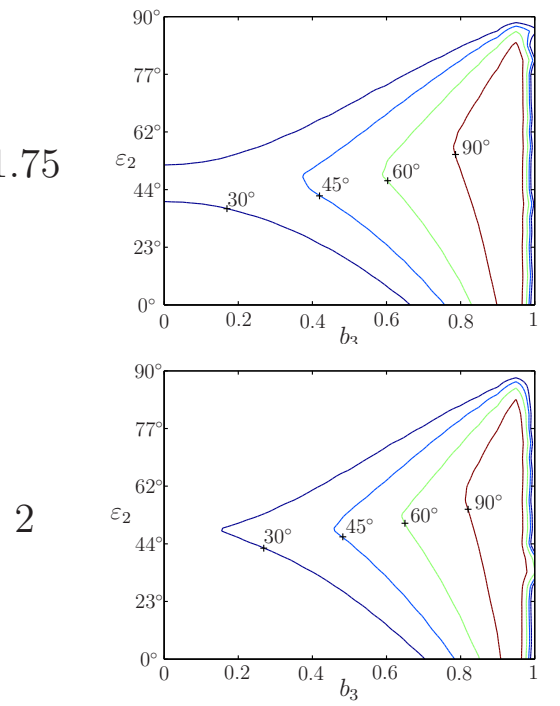
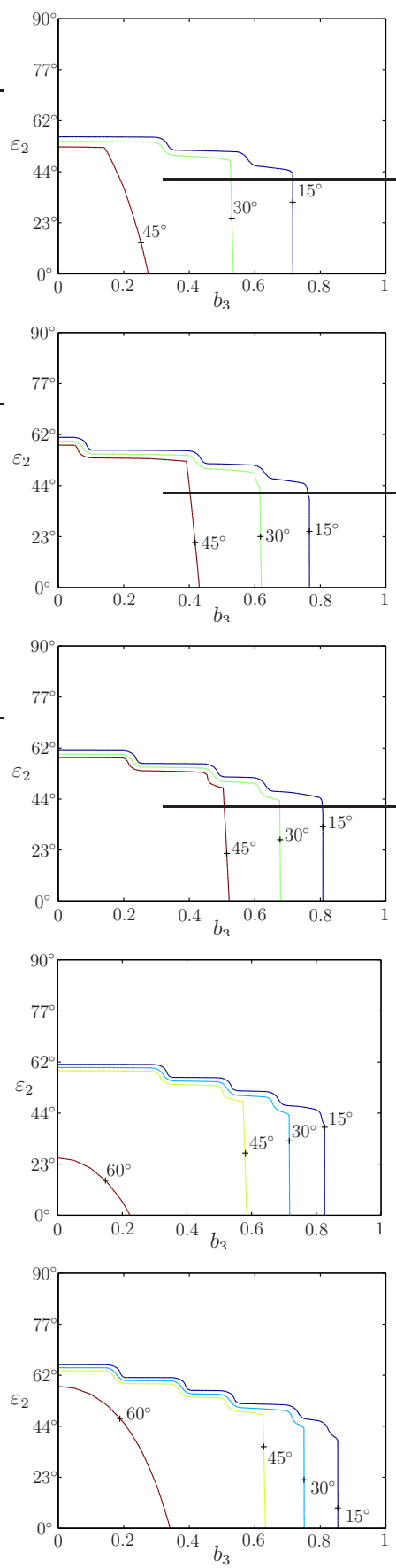
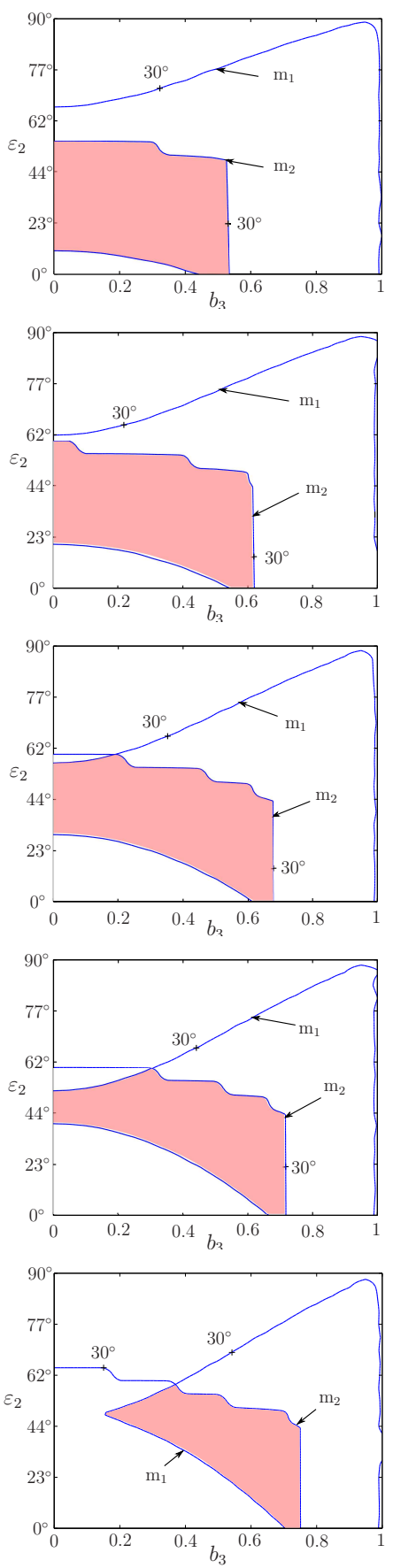


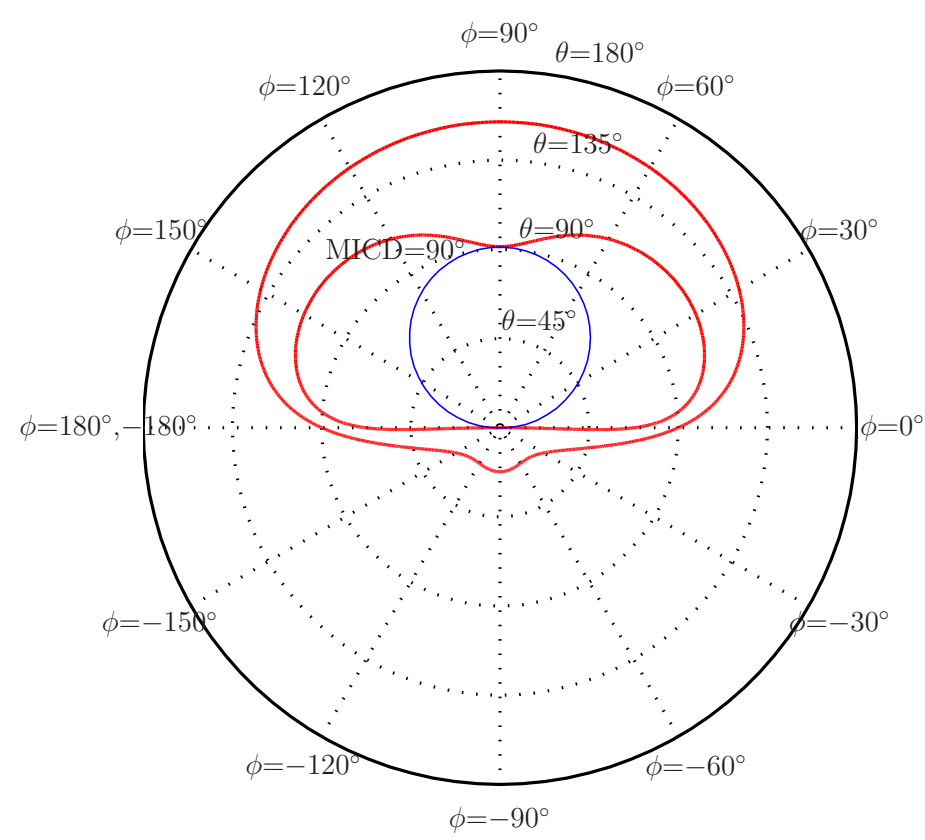

Figure 9: Maximum Inscribed Circle Diameter

For the parallel manipulator shown in Fig. 1, MICD is traced when the moving-platform works at five different heights, namely $Z=1,1.25,1.5,1.75,2$. The distributions of MICD in both operation modes $x_{1}=0$ and $x_{2}=0$ (denoted by $\mathrm{m}_{1}$ and $\mathrm{m}_{2}$, respectively) are provided in Table 2.

In operation mode $x_{1}=0$, the maximum MICD that can be reached is up to $90^{\circ}$. The higher $Z$, the smaller MICD. MICD is a maximum when $Z=1$ and the moving-platform size is 0.6 times smaller than the base. MICD is a minimum when $Z=2$ and when the moving-platform is almost as large as the base $\left(0.82 \leq b_{3} \leq 0.98\right)$. As a consequence, many architectures of 3 -RPS parallel manipulator will perform greater value of MICD in operation mode $x_{1}=0$ if it is operated at lower altitude.

In operation mode $x_{2}=0$, the maximum MICD which can be attained by all designs at $Z=1.75$ up to $Z=2$ is only up to $60^{\circ}$. At the lower altitude, i.e. $Z=1,1.25,1.5$, MICD which can be reached by the platform is only up to $45^{\circ}$. Unlike in operation mode $x_{1}=0$, the region of MICD in operation mode $x_{2}=0$ increases significantly as the altitude increases. As a consequence, many architectures of 3-RPS parallel manipulator will perform greater value of MICD in operation mode $x_{2}=0$ if it is operated at higher altitude.

For the manipulator to be used as an ankle rehabilitation device, the minimum tilting capability which should be performed by the moving-platform in both operation modes $x_{1}=0$ and $x_{2}=0$ is $30^{\circ}$, as explained in Sec. 2.2. Thus, a constraint is defined as follows:

$$
\mathrm{MICD} \geq 30^{\circ}
$$


The optimum region satisfying Eq. (27) is derived at each height which is shown by the red surfaces in Table 2 .

The optimum regions shown in Table 2 decrease as the altitude increases. At lowest altitude $(Z=1)$, many architectures of 3-RPS parallel manipulator can exhibit at least $30^{\circ}$ of MICD in both operation modes. It occurs if the moving-platform dimension is a lot smaller than the base $\left(b_{3} \leq 0.5\right)$. At highest altitude $(Z=2)$, very few architectures of 3-RPS parallel manipulator can perform at least $30^{\circ}$ of MICD in both operation modes.

All optimum regions are combined as shown in Fig. 10 and a new optimum region is obtained as shown by grey surface. This region tells us the set of optimum design parameters $b_{3}, \varepsilon_{2}$ that satisfies Eq. (27) at five different heights simultaneously. It is noticeably that this optimum region lies below $\varepsilon_{2} \approx 53^{\circ}$. It means that the second and third legs should be assembled at maximum angle of $53^{\circ}$ from the $x$-axis.

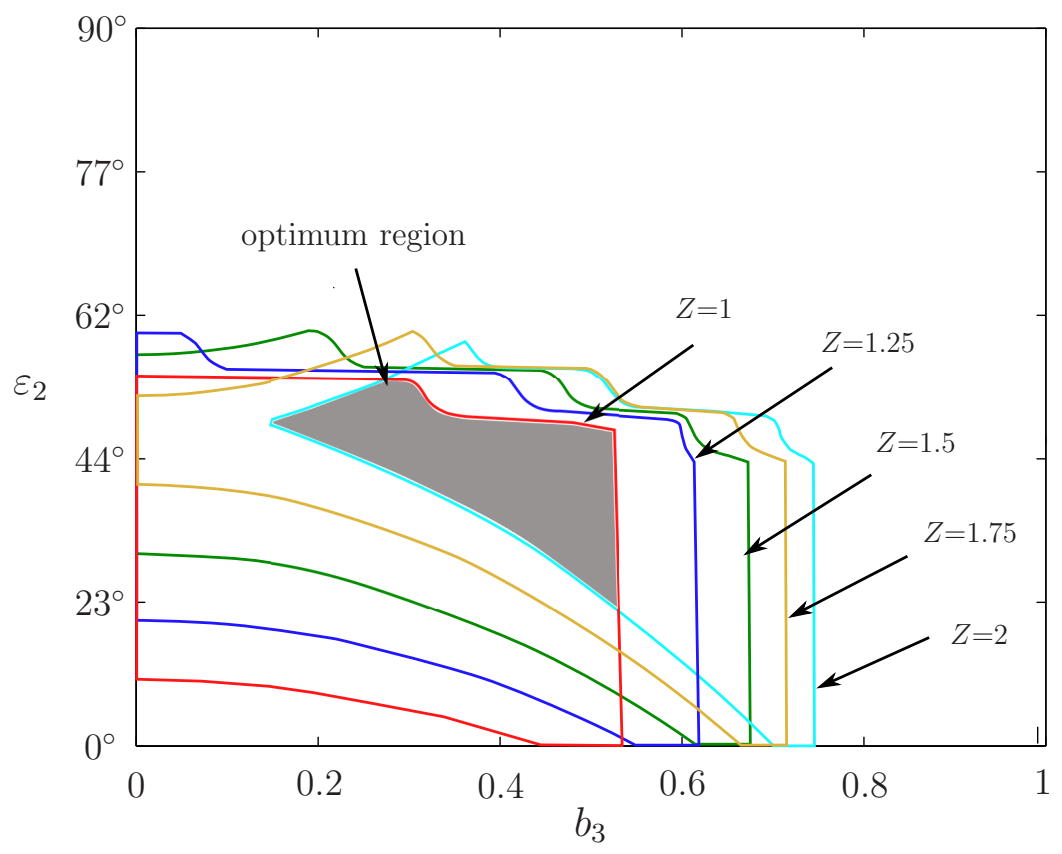

Figure 10: Optimum set of design parameters when MICD $\geq 30^{\circ}$

\section{$6 \quad$ Reconfiguration strategy}

One selected architecture of 3-RPS parallel manipulator from the optimum region shown in Fig. 10, can work at minimum tilt angle of $30^{\circ}$ from $Z=1$ up to $Z=2$. However, if the moving-platform is required to work at lower and higher altitudes, i.e. $Z \leq 1$ and $Z \geq 2$ respectively, its MICD may reduce below $30^{\circ}$ in either operation mode $x_{1}=0$ or operation mode $x_{2}=0$. To overcome this issue, the moving-platform should switch from operation mode $x_{1}=0$ to $x_{2}=0$ or vice versa, depending on the orientation capability of each operation mode. This reconfiguration should be conducted at a specific range of height. 
Let us consider the parameters $b_{3}=0.5$ and $\varepsilon_{2}=44^{\circ}$, which are selected within the optimum region given in Fig. 10. By substituting these values into the design condition $2 \mathrm{C}$ defined in Eq. (11) and Eq. (25), other design parameters can be derived as shown in Table 3.

Table 3: Selected design parameters

\begin{tabular}{ccccccccc}
\hline \hline \multicolumn{3}{c}{ Base } & \multicolumn{4}{c}{ Platform } & \multicolumn{3}{c}{ Angles } \\
$a_{1}$ & $a_{2}$ & $a_{3}$ & $b_{1}$ & $b_{2}$ & $b_{3}$ & $\varepsilon_{1}$ & $\varepsilon_{2}$ & $\varepsilon_{3}$ \\
\hline 1.38 & 1 & 1 & 0.69 & 0.5 & 0.5 & $0^{\circ}$ & $44^{\circ}$ & $-44^{\circ}$ \\
\hline \hline
\end{tabular}

To investigate the relationship between MICD and the height $Z$ of this manipulator, all MICD is plotted as a function of moving-platform height from 0 up to 5, as shown in Fig. 11. Let us assume that the selected 3-RPS parallel manipulator works from altitude 0 to 5 with minimum orientation defined by Eq. (27), namely $\theta_{\min }=30^{\circ}$, shown by dash line in Fig. 11 .

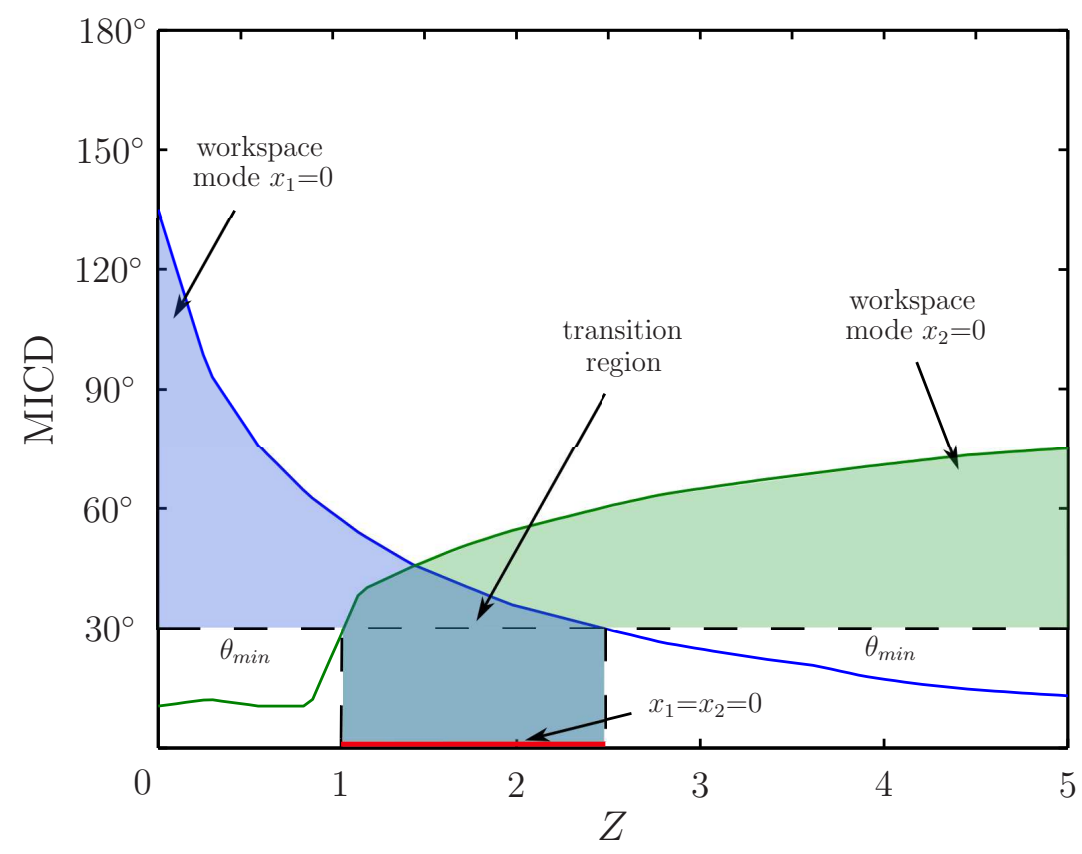

Figure 11: Reconfiguration altitude

The workspaces of operation modes $x_{1}=0$ and $x_{2}=0$ can be detected as shown by the blue and green surfaces, respectively. It is shown that the orientation workspace limited by MICD of operation mode $x_{1}=0$ is much larger at lower altitude, i.e. $Z \leq 2.5$. For operation mode $x_{2}=0$, the orientation workspace limited by MICD is much larger at higher altitude, i.e. $Z \geq 1$, although MICD cannot reach up to $90^{\circ}$. The intersection of the two workspaces yield a transition region that allows the manipulator to switch from operation mode $x_{1}=0$ to $x_{2}=0$ or vice versa, while maintaining high orientation capability given in Eq. (27). This transition region is bounded between $1 \leq Z \leq 2.5$. 
For the manipulator to be able to work at $Z \geq 2.5$ while maintaining the orientation to be $\theta \geq 30^{\circ}$, the moving-platform should switch into operation mode $x_{2}=0$. To switch the operation mode, the condition of constraint singularity defined in Eq. (22) should be satisfied. It means that the moving-platform should enter a constraint singularity configuration by changing its orientation to be $\theta=0^{\circ}$. It can be carried out if and only if the moving-platform reaches the transition region, namely when the moving-platform height is in the range $1 \leq Z \leq 2.5$. The constraint singularity configuration is described as a common configuration that belongs to both operation modes simultaneously, which yields $x_{1}=x_{2}=0$ (shown by red line in Fig. 11).

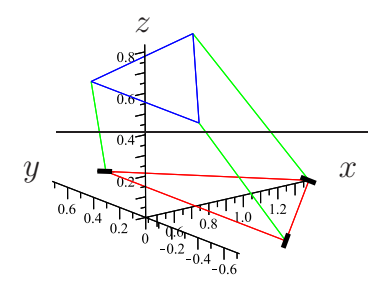

(a) Mode $x_{1}=0$ at $Z<1$

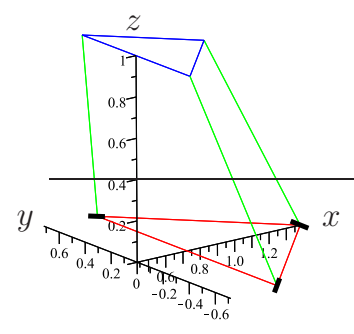

(b) Reconfiguration at $Z=1$

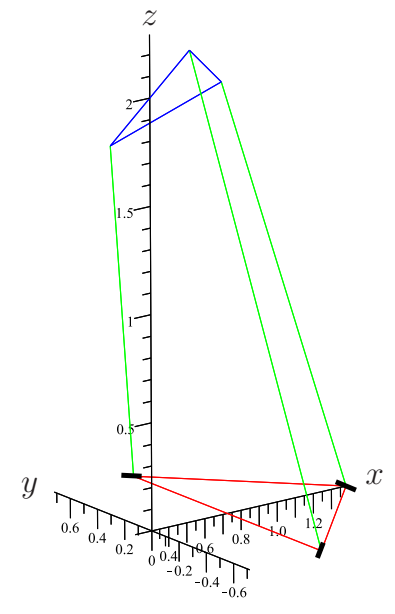

(c) Mode $x_{2}=0$ at $Z>1$

Figure 12: Reconfiguration strategy to maintain $\theta_{\min }=30^{\circ}$ at different heights

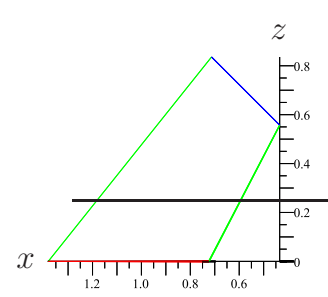

(a) Mode $x_{1}=0$ at $Z<1$

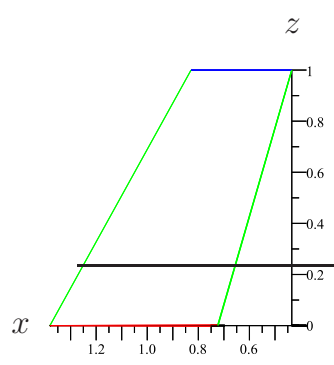

(b) Reconfiguration at $Z=1$

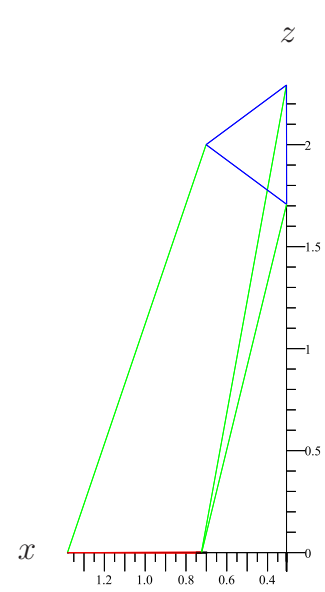

(c) Mode $x_{2}=0$ at $Z>1$

Figure 13: Side view: reconfiguration strategy to maintain $\theta_{\min }=30^{\circ}$ at different heights

Once the constraint singularity configuration is reached, the moving-platform can enter the operation mode $x_{2}=0$ by switching its orientation back to $\theta \geq 30^{\circ}$. Eventually, the movingplatform can work with minimum orientation $\theta \geq 30^{\circ}$ at higher altitude. All steps and poses of 
the reconfiguration strategy are demonstrated in Figs. 12(a)-12(c). Figs. 13(a)-13(c) illustrate manipulator poses during reconfiguration strategy from side view. This reconfiguration strategy can be executed easily and is not affected by the leg collisions since the transition configuration occurs inside the workspace at $\theta=0^{\circ}$.

\section{Conclusions}

In this paper, several constraint-based design conditions were introduced for the design of 3-RPS parallel manipulators. Those conditions describe the relations among the base and movingplatform design parameters. One design condition was selected and by following this condition, a 3-RPS parallel manipulator with 3-dof operation mode was generated and used as an ankle rehabilitation device. The kinematic optimization was performed to synthesize its optimum parameters. First, the parametrization of orientation workspace was performed to investigate the moving-platform capability in terms of translational and rotational motions. It reveals that the orientation workspace is not symmetrical. A performance index, named Maximum Inscribed Circle Diameter (MICD) was proposed to trace the maximum tilt angle for any azimuth angle without reaching any singularity. A two-dimensional design space was created which to make it possible to plot the distributions of MICD for different moving-platform heights. The optimum regions with regard to MICD were obtained and a set of dimensionless design parameters was selected within this region. The relationship among MICD, operation modes and moving-platform height was investigated for the selected design. It shows that the evolution of MICD as a function of moving-platform height is the opposite. To overcome this issue, a reconfiguration strategy was proposed to ensure the moving-platform working above the minimum required orientation for any moving-platform height.

\section{References}

\section{References}

[1] K. H. Hunt. "Structural Kinematics of in-parallel-actuated Robot-arms", Journal of Mechanisms, Transmission, and Automation in Design, 105, pp. 705-712, 1983.

[2] J. Schadlbauer, D.R. Walter, M. Husty. "The 3-RPS Parallel Manipulator from an Algebraic Viewpoint", Mechanism and Machine Theory, 75, pp. 161-176, 2014.

[3] M. Husty, M. Pfurner, H-P. Shröcker, K. Brunnthaler. "Algebraic Methods in Mechanism Analysis and Synthesis", Robotica, 25(6), pp. 661-675, 2007.

[4] M. Husty, M. Pfurner, H-P. Shröcker. "A New and Efficient Algorithm for the Inverse Kinematics of a General Serial 6R Manipulator", Mechanism and Machine Theory, 42 (1), pp. 66-81, 2007.

[5] M. Pfurner, T. Stigger, M. Husty. "Algebraic Analysis of Over-constrained Single Loop Four Link Mechanisms with Revolute and Prismatic Joints", Mechanism and Machine Theory, 114, pp. 11-19, 2017. 
[6] Z. Chen, H. Ding, W. Cao, Z. Huang. "Axodes Analysis of the Multi DOF Parallel Mechanisms and Parasitic Motion", in ASME International Design Engineering Technical Conferences and Computers and Information in Engineering Conference,Portland, Oregon, USA, 2013.

[7] J. Schadlbauer, L. Nurahmi, M. Husty, P. Wenger, S. Caro. "Operation Modes in LowerMobility Parallel Manipulators", In: Kecskeméthy, A and Geu Flores, F, Interdisciplinary Applications of Kinematics, 26, Springer, pp. 1-9, 2013.

[8] D. Gan, J. Dias, L. Seneviratne. "Unified Kinematics and Optimal Design of a 3-rRPS Metamorphic Parallel Mechanism with a Reconfigurable Revolute Joint", Mechanism and Machine Theory, 96, pp. 239-254, 2016.

[9] Z. Huang, Y. Huang. "Kinematic Characteristics Analysis of 3-DOF In-Parallel Actuated Pyramid Mechanism", Mechanism and Machine Theory, 31 (8), pp. 1009-1018, 1996.

[10] Z. Chen, W.-A. Cao, Z. Huang. "Type Synthesis of 3-DOF Rotational Parallel Mechanisms With No Intersecting Axes", ASME Paper No. DETC2012-70846, 2012.

[11] L. Nurahmi, J. Schadlbauer, S. Caro, M. Husty, P. Wenger. "Kinematic Analysis of 3-RPS Cube Parallel Manipulator", Journal of Mechanisms and Robotics, 7, pp. 0110081-011008-11, 2015.

[12] R. Kalla, L. Nurahmi, S. Bandyopadhay, S. Caro, P. Wenger. "A Study of $\Sigma^{2}$ Singularities in the 3-RPS Parallel Manipulator", In: 2nd International and 17th National Conference on Machines and Mechanisms, India, December 16-19, 2015.

[13] A. Nayak, L. Nurahmi, P. Wenger, S. Caro. "Comparison of 3-RPS and 3-SPR Parallel Manipulators Based on Their Maximum Inscribed Singularity-Free Circle", In: New Trends in Mechanism and Machine Science, pp. 121-130, 2017.

[14] S. Maraje, L. Nurahmi, S. Caro. "Operation Modes Comparison of a Recongurable 3PRS Parallel Manipulator Based on Kinematic Performance", In: ASME International Design Engineering Technical Conferences and Computers and Information in Engineering Conference, Charlotte, NC, USA, 2016.

[15] X-J. Liu, I. Bonev. "Orientation Capability, Error Analysis, and Dimensional Optimization of Two Articulated Tool Heads with Parallel Kinematics", Journal of Manufacturing Science and Engineering, 130, pp. 011015-1-011015-9, 2008.

[16] D. M. Gan, J. S. Dai, J. Dias, R. Umer, L. D. Seneviratne, "Singularity-Free Workspace Aimed Optimal Design of a 2T2R Parallel Mechanism for Automated Fiber Placement", ASME Journal of Mechanisms and Robotics, 7(4), pp. 041022-1-041022-9, 2015.

[17] D. M. Gan, J. S. Dai, J. Dias, L. D. Seneviratne, "Forward Kinematics Solution Distribution and Analytic Singularity-Free Workspace of Linear-Actuated Symmetrical Spherical Parallel Manipulators", ASME Journal of Mechanisms and Robotics, 7(4), pp. 041007-1041007-8, 2015. 
[18] R. S. Ball. "A Treatise on the Theory of Screws", Cambridge University Press, Cambridge, 1900.

[19] G. Sutherland, B. Roth. "A Transmission Index for Spatial Mechanisms", Journal of Engineering for Industry, 95 (2), pp. 589-597, 1973.

[20] C. Chen, J. Angeles. "Generalized Transmission Index and Transmission Quality for Spatial Linkages", Mechanism and Machine Theory, 42 (9), pp. 1225-1237, 2007.

[21] X-J. Liu, X. Chen, M. Nahon. "Motion/Force Constrainability Analysis of Lower-Mobility Parallel Manipulators", Journal of Mechanisms and Robotics, 6 (3), pp. 0310061-0310069, 2014.

[22] H. Liu, T. Huang, A. Kecskeméthy, D. G. Chetwynd. "A Generalized Approach for Computing the Transmission Index of Parallel Mechanisms", Mechanism and Machine Theory, 74, pp. 245-256, 2014.

[23] F. Xie, X-J. Liu, J. Wang, M. Wabner. "Kinematic Optimization of a Five Degrees-ofFreedom Spatial Parallel Mechanism with Large Orientational Workspace", Journal of Mechanisms and Robotics, 9, pp. 051005-1-051005-9, 2017.

[24] D. M. Gan, J. S. Dai, J. Dias, L. D. Seneviratne, "Variable Motion/Force Transmissibility of a Metamorphic Parallel Mechanism with Reconfigurable 3T and 3R Motion", ASME Journal of Mechanisms and Robotics, 8(5), pp. 051001-1-051001-9, 2016.

[25] L. Nurahmi, S. Caro, P. Wenger. "Design of 3-RPS Parallel Manipulator based on Operation Mode", in 14th IFToMM World Congress in Mechanism and Machine Science. Taipei, Taiwan, 2015.

[26] L. Nurahmi, M. Solichin, D. Harnany, A. Kurniawan. "Dimension Synthesis of 3-RPS Parallel Mechanism with Intersecting R-axes for Ankle Rehabilitation Device", In: 18th International Conference on Advanced Robotics, pp. 269-274, 2017.

[27] L. Nurahmi, M. Solichin. "Motion Type of 3-RPS Parallel Manipulator for Ankle Rehabilitation Device", Proceeding ICAMIMIA 2017: International Conference on Advanced Mechatronics, Intelligent Manufacture, and Industrial Automation, pp. 74-79, 2017.

[28] Q. Li, Z. Chen, Q. Chen, C. Wu, X. Hu. "Parasitic Motion Comparison of 3-PRS Parallel Mechanism with Different Limb Arrangements", Robotics and Computer-Integrated Manufacturing, 27 (2), pp. 389-396, 2011.

[29] R. Lin, W. Guo, F. Gao. "On Parasitic Motion of Parallel Mechanisms", in ASME International Design Engineering Technical Conferences and Computers and Information in Engineering Conference, Charlotte, NC, USA, 2016.

[30] W. Ye, L. He, Q. Li. "A New Family of Symmetrical 2T2R Parallel Mechanisms Without Parasitic Motion", Journal of Mechanisms Robotics, 10 (1), pp. 011006-011006-9, 2017.

[31] I. A. Bonev. "Geometric Analysis of Parallel Mechanisms", Doctoral dissertation, University of Laval, Quebec, QC, Canada, 2002. 
[32] I. A. Bonev. "Direct Kinematics of Zero-Torsion Parallel Mechanisms", in IEEE International Conference on Robotics and Automation (ICRA), Pasadena, CA, May 1923, pp. 3851-3856, 2008.

[33] S. Amine, O. Mokhiamar, S. Caro. "Classification of 3T1R Parallel Manipulators Based on Their Wrench Graph", Journal of Mechanisms and Robotics, 9 (1), pp. 011003-011003-10, 2017.

[34] S. Amine, L. Nurahmi, P. Wenger, S. Caro. "Conceptual Design of Schoenflies Motion Generators Based on the Wrench Graph", ASME International Design Engineering Technical Conferences and Computers and Information in Engineering Conference, 6A, 37th Mechanisms and Robotics Conference, 2013.

[35] S. Caro, W. A. Khan, D. Pasini, J. Angeles, "The Rule-based Conceptual Design of the Architecture of Serial Schnflies-motion Generators", Mechanism and Machine Theory, 45 (2), pp. 251-260, 2010.

[36] W. A. Khan, S. Caro, J. Angeles, D. Pasini, "A Formulation of Complexity-Based Rules for the Preliminary Design Stage of Robotic Architectures", International Conference on Engineering Design, ICED07, Paris, France, 2007. 